\title{
Collapsing domain walls in the two-Higgs-doublet model and deep insights from the EDM
}

\author{
Ning Chen, ${ }^{a}$ Tong $\mathbf{L i}^{a}{ }^{a}$ Zhaolong Teng ${ }^{a}$ and Yongcheng $\mathbf{W} \mathbf{u}^{b}$ \\ a School of Physics, Nankai University, \\ Tianjin 300071, China \\ ${ }^{b}$ Ottawa-Carleton Institute for Physics, Carleton University, \\ 1125 Colonel By Drive, Ottawa, Ontario K1S 5B6, Canada \\ E-mail: chenning_symmetry@nankai.edu.cn, litong@nankai.edu.cn, \\ tengcl@mail.nankai.edu.cn, ycwu@physics.carleton.ca
}

ABSTRACT: We study the domain wall solutions in the general two-Higgs-doublet model (2HDM) with a CP-violating phase. The $2 \mathrm{HDM}$ with the spontaneouse $\mathrm{CP}$ violation is found to have domain wall solutions whose tensions are $\mathcal{O}\left(10^{6}\right) \mathrm{GeV}^{3}$, which are excluded by the Zel'dovich-Kobzarev-Okun bound. With the explicit CP-violating (CPV) terms as the so-called biased term in the scalar potential, domain walls can collapse in the early Universe. The sizes of the explicit CP violation can be constrained from the Big Bang nucleosynthesis. This constraint is converted to the CPV mixing of $\alpha_{c}$, and is mostly sensitive to the mass splittings between two heavy neutral Higgs bosons. We estimate the possible gravitational wave signals and the electric dipole moment (EDM) predictions due to the domain wall collapsing. It turns out that the peak spectrum of the $\mathrm{GW}$ from the domain wall collapsing cannot be probed in any future program. In contrast, the untenable regions with very tiny explicit CPV parameter in the Higgs potential has been partially excluded by the latest electron EDM measurements at the ACME-II and will be further confirmed or excluded by the future ACME-III projection.

Keywords: CP violation, Discrete Symmetries, Cosmology of Theories beyond the SM

ARXIV EPRINT: 2006.06913 


\section{Contents}

1 Introduction 1

2 The general 2HDM with the CPV 3

2.1 The 2HDM potential 3

2.2 The 2HDM potential with the SCPV 4

2.3 The 2HDM potential with the ECPV 6

$\begin{array}{lll}2.4 & \text { The unitarity and stability constraints } & 7\end{array}$

3 Domain walls in the CPV 2HDM $\quad 8$

3.1 The domain wall solutions 8

$\begin{array}{ll}3.2 & \text { The biased term from the ECPV } \\ \end{array}$

$\begin{array}{ll}3.3 \text { The cosmological constraints } & 11\end{array}$

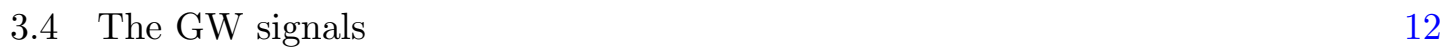

4 The EDM measurements 13

5 Conclusion and discussion $\quad 17$

A The mass spectrum and self couplings with the SCPV and the ECPV 18

$\begin{array}{ll}\text { A.1 The mass spectrum and self couplings with the SCPV } & 18\end{array}$

$\begin{array}{ll}\text { A.2 The mass spectrum and self couplings with the ECPV } & 21\end{array}$

A.3 The Yukawa couplings in the CPV 2HDM 24

\section{Introduction}

The Standard Model (SM) of particle physics has been experimentally verified to be successful, with the discovery of the $125 \mathrm{GeV}$ Higgs boson [1, 2] in 2012. Yet, the SM itself cannot address the long-lasting puzzle of the baryon asymmetry in the Universe (BAU). Thus, many efforts have been made to realize the three Sakharov conditions [3] by extending the SM. It is quite general that the extensions to the SM lead to larger symmetries. During the phase transitions of the early Universe, different symmetry breaking patterns are likely to produce topological defects. This is known as the Kibble-Zurek mechanism [46]. The domain walls, which are two-dimensional topological defects, can exist when the 0 -th homotopy group associated with the symmetry breaking of $\mathcal{G} \rightarrow \mathcal{H}$ is nontrivial, i.e., $\Pi_{0}(\mathcal{G} / \mathcal{H}) \neq 1$. The existence of the domain wall solutions in any new physics can be problematic, since their energy density scale as $t^{-1}$ with respect to time $t$. This means once they are formed in the early Universe, they can dominate the energy densities over radiation and matter and thus spoil the standard cosmology. 
In this work, we study the possible domain wall solutions arising from the $\mathrm{CP}$ symmetries of the two-Higgs-doublet model (2HDM). Originally, the study of the electroweak theory with two Higgs doublets in the scalar sector was motivated to achieve the spontaneous CP violations (SCPV) [7]. However, the existence of two degenerate vacua with opposite-sign CP phases can lead to the domain wall solutions. The domain wall solutions in the 2HDM were previously studied in refs. [8-10], and recently revisited in refs. [11, 12]. With the typical energy scale of few hundred GeV for the heavy scalars, the tensions of the stable domain walls in the $2 \mathrm{HDM}$ are found of the size $\sigma \sim \mathcal{O}\left(10^{6}\right) \mathrm{GeV}^{3}$, which is excluded by the Zel'dovich-Kobzarev-Okun bound [13].

Given the possible domain wall solutions in the CPV 2HDM, let us briefly review the existing solutions. The first well-known method in the new physics model building is to invoke an inflation phase [14] during the domain wall formation. However, the inflation models typically operate at the GUT scale of $\Lambda_{\mathrm{GUT}} \sim 10^{16} \mathrm{GeV}$, which does not seem to remedy the SCPV domain wall forming at the electroweak scale. Note that there exist other possibilities to solve the domain wall problem if some specific global symmetry is spontaneously broken in a post inflation epoch. For the Peccei-Quinn symmetry solving the strong CP problem in QCD, one solution is the so-called Lazarides-Shafi mechanism [15] in which the degenerate vacua are connected to each other by another continuous group transformation. The choice of the symmetry group and Higgs representations in this mechanism has to be cautious, which makes the possibility difficult to happen [16]. If the global lepton number symmetry is spontaneously broken, one can introduce auxiliary Majorana field with non-trivial $\mathrm{SU}(2)_{\mathrm{L}}$ quantum number in Majoron model or a supersymmetric context to solve the domain wall problem [17]. One possible way to evade the domain wall problem is to include a small symmetry breaking term, that is the so-called biased term in the Higgs potential. In this perspective, the symmetry of the CP transformation is approximate. With such a mechanism [18-20], the possible domain walls were unstable and collapsed before they overclose the Universe. Indeed, we found that such biased terms are nothing but the explicit CPV (ECPV) parameters in the 2HDM potential.

With the ECPV parameters as the biased terms in the $2 \mathrm{HDM}$ potential, one may expect gravitational wave (GW) signals associated with the domain wall collapses. This was previously discussed in refs. [21-26]. Therefore, it is natural to ask if such GW signals can be probed at the future programs, such as the satellite-based interferometers of LISA [27, 28], Taiji [29], and Tianqin [30], or the radio telescope of square kilometer arrays (SKA) [31] and the Japanese space GW antenna (DECIGO) [32]. Note that such GW signals are different from those produced due to the bubble collisions during the strongly first-order phase transitions. The constraint on the ECPV parameters is imposed so that the corresponding biased terms are sufficiently large to collapse the domain walls before the epoch of the big bang nucleosynthesis (BBN). In ref. [25], we found such signals can be sufficiently strong when the corresponding new physics scale is above about $10 \mathrm{TeV}$. However, as we shall show, the related GW peak spectrum here is typically $\lesssim \mathcal{O}\left(10^{-23}\right)$ and below the future search sensitivities of SKA [31] and DECIGO [32]. Alternatively, the precise measurements of the electric dipole moments (EDM) play an intriguing role in constraining the small ECPV parameters. The ongoing and upcoming EDM measurements include the electron EDM (eEDM) from the ACME collaboration [33, 34], the mercury 
EDM [35], and the radium EDM [36]. The EDM measurements are usually interpreted to constrain CPV mixings in the context of the 2HDM [37-45], or the SUSY-breaking scale [46]. Usually, the constraint on CP-violating 2HDM from EDM was performed in the absence of spontaneous CP-violating phase to avoid the problematic SCPV domain walls [38]. With the future improvements of the eEDM precision measurements from the ACME-III, given a few hundred GeV heavy scalar masses, we find that they can be used to exclude sizable regions of the ECPV parameters in the 2HDM potential jointly with the $\mathrm{BBN}$ constraint. In this sense, the EDM experiments provide us deep insights to the very tiny ECPV term that triggered the domain wall collapse in the early Universe. This is completely different from what was usually discussed in the context of SUSY, such that the precision measurements of the EDM will constrain the corresponding new physics scale above $\mathcal{O}(10) \mathrm{TeV}[46]$.

The rest of the paper is organized as follows. In section 2, we review the general 2HDM. We focus on the minimization conditions and the mass spectrum in both the SCPV and the ECPV scenarios. The parameters between the generic basis and the physical basis are built for solving the domain wall. The theoretical constraints of the perturbative unitarity and the vacuum stabilities are imposed to the scalar self couplings. In section 3 , we present the domain wall solutions in the CPV 2HDM. The domain walls from the SCPV typically have tensions of $\mathcal{O}\left(10^{6}\right) \mathrm{GeV}^{3}$, which should be collapsed with sufficiently sizable biased terms. The ECPV parameter in the $2 \mathrm{HDM}$, which we choose to be $\operatorname{Im} \lambda_{5}$, will be bounded from below by considering the BBN constraint. By estimating the corresponding GW signals from the domain wall collapses, they are beyond the search sensitivities of any future satellite-based observation programs. In section 4, we turn to the estimations of the eEDM in the domain wall collapsing scenario. The current and the future projections of the eEDM measurements from the ACME are used to set upper limits to the CPV mixing angle, as well as exclude the ECPV parameter directly. We summarize our results in section 5. In the appendix A, we provide details of deriving the 2HDM mass spectrum for both the SCPV and the ECPV scenarios. Besides, the Yukawa couplings for the CPV $2 \mathrm{HDM}$ are also presented therein.

\section{The general 2HDM with the CPV}

In this section, we review the general CP-violating $2 \mathrm{HDM}$ and focus on the global symmetry from the $\mathrm{CP}$ transformation of the potential. Previous studies of the symmetries and topological structures of the $2 \mathrm{HDM}$ include refs. [11, 38, 47-53]. Our focus will be on the $\mathrm{CP}$ symmetries in the $2 \mathrm{HDM}$ potential, with the details of deriving the relative $\mathrm{CP}$ violating phase of $\theta$ given in section A.1 and A.2 for the SCPV and the ECPV scenarios, respectively. The Yukawa couplings for the CPV 2HDM are listed in section A.3.

\subsection{The 2HDM potential}

We write down the $2 \mathrm{HDM}$ potential with the softly broken $\mathbb{Z}_{2}$ symmetry as

$$
\begin{aligned}
V\left(\Phi_{1}, \Phi_{2}\right)= & m_{11}^{2}\left|\Phi_{1}\right|^{2}+m_{22}^{2}\left|\Phi_{2}\right|^{2}-\left(m_{12}^{2} \Phi_{1}^{\dagger} \Phi_{2}+\text { h.c. }\right)+\frac{\lambda_{1}}{2}\left|\Phi_{1}\right|^{4}+\frac{\lambda_{2}}{2}\left|\Phi_{2}\right|^{4} \\
& +\lambda_{3}\left|\Phi_{1}\right|^{2}\left|\Phi_{2}\right|^{2}+\lambda_{4}\left(\Phi_{1}^{\dagger} \Phi_{2}\right)\left(\Phi_{2}^{\dagger} \Phi_{1}\right)+\left[\frac{\lambda_{5}}{2}\left(\Phi_{1}^{\dagger} \Phi_{2}\right)^{2}+\text { h.c. }\right]
\end{aligned}
$$


Here, $\left(m_{12}^{2}, \lambda_{5}\right)$ are complex for the CPV case, and we parametrize them by $m_{12}^{2} \equiv$ $\left|m_{12}^{2}\right| e^{i \delta_{1}}=\operatorname{Re} m_{12}^{2}+i \operatorname{Im} m_{12}^{2}$ and $\lambda_{5} \equiv\left|\lambda_{5}\right| e^{i \delta_{2}}=\operatorname{Re} \lambda_{5}+i \operatorname{Im} \lambda_{5}$. All parameters are real for the CP-conserving (CPC) case. To study the domain wall solution of the $2 \mathrm{HDM}$ under the CP transformations below, we follow the refs. $[10,53,54]$ to parametrize two Higgs doublets as follows

$$
\Phi_{1}=\left(\begin{array}{c}
H_{1}^{+} \\
\frac{1}{\sqrt{2}}\left(\varphi_{1}+H_{1}^{0}+i A_{1}^{0}\right)
\end{array}\right), \quad \Phi_{2}=e^{i \Theta}\left(\begin{array}{c}
H_{2}^{+} \\
\frac{1}{\sqrt{2}}\left(\varphi_{2}+H_{2}^{0}+i A_{2}^{0}\right)
\end{array}\right)
$$

In order to obtain the domain wall solutions below, we treat $\left(\varphi_{1}, \varphi_{2}, \Theta\right)$ as background fields. When the electroweak symmetry breaking (EWSB) occurred, they obtain the vacuum expectation values (VEVs) as $\left\langle\varphi_{1,2}\right\rangle=v_{1,2},\langle\Theta\rangle=\theta$, with $\theta$ to be solved below for both the SCPV and the ECPV scenarios. As usual, two VEVs are parametrized as $v_{1}=v c_{\beta}$ and $v_{2}=v s_{\beta} .{ }^{1}$ The minimizations of the most general 2HDM poential (2.1) are the following

$$
\begin{aligned}
m_{11}^{2} & =\frac{t_{\beta}}{c_{\theta}} \operatorname{Re} m_{12}^{2}-\frac{1}{2} \lambda_{1} v^{2} c_{\beta}^{2}-\frac{1}{2}\left(\lambda_{3}+\lambda_{4}+\operatorname{Re} \lambda_{5}-\operatorname{Im} \lambda_{5} t_{\theta}\right) v^{2} s_{\beta}^{2}, \\
m_{22}^{2} & =\frac{1}{t_{\beta} c_{\theta}} \operatorname{Re} m_{12}^{2}-\frac{1}{2} \lambda_{2} v^{2} s_{\beta}^{2}-\frac{1}{2}\left(\lambda_{3}+\lambda_{4}+\operatorname{Re} \lambda_{5}-\operatorname{Im} \lambda_{5} t_{\theta}\right) v^{2} c_{\beta}^{2}, \\
\operatorname{Re} m_{12}^{2} s_{\theta}+\operatorname{Im} m_{12}^{2} c_{\theta} & =\frac{1}{2}\left(\operatorname{Re} \lambda_{5} s_{2 \theta}+\operatorname{Im} \lambda_{5} c_{2 \theta}\right) v^{2} s_{\beta} c_{\beta} .
\end{aligned}
$$

In the special case of vanishing spontaneous CPV phase $\theta=0$, the minimization conditions are reduced to

$$
\begin{aligned}
m_{11}^{2} & =\operatorname{Re}_{12}^{2} t_{\beta}-\frac{1}{2} \lambda_{1} v^{2} c_{\beta}^{2}-\frac{1}{2}\left(\lambda_{3}+\lambda_{4}+\operatorname{Re} \lambda_{5}\right) v^{2} s_{\beta}^{2}, \\
m_{22}^{2} & =\operatorname{Re} m_{12}^{2} / t_{\beta}-\frac{1}{2} \lambda_{2} v^{2} s_{\beta}^{2}-\frac{1}{2}\left(\lambda_{3}+\lambda_{4}+\operatorname{Re} \lambda_{5}\right) v^{2} c_{\beta}^{2}, \\
\operatorname{Im} m_{12}^{2} & =\frac{1}{2} \operatorname{Im} \lambda_{5} v^{2} s_{\beta} c_{\beta} .
\end{aligned}
$$

\subsection{The 2HDM potential with the SCPV}

In a general $2 \mathrm{HDM}$, the CP symmetry is conserved only if three invariants of $J_{1,2,3}$ defined in refs. [47, 48,53] are all real. This can be realized with two possibilities, namely, either with

$$
\text { SCPV1 : } \operatorname{Im}\left[\left(m_{12}^{2}\right)^{2} \lambda_{5}^{*}\right]=0
$$

or

$$
\operatorname{SCPV} 2: \lambda_{1}=\lambda_{2}, \quad m_{11}^{2}=m_{22}^{2}, \quad t_{\beta}=1 .
$$

In this work, we focus on the SCPV1 scenario defined in ref. [53], and use the notion of SCPV throughout the context. Thus, it is equivalent to take $2 \delta_{1}-\delta_{2}=n \pi$, with $n$ being an

\footnotetext{
${ }^{1}$ Throughout the context, we use the short-handed notations of $s_{\beta} \equiv \sin \beta$ and $c_{\beta} \equiv \cos \beta$.
} 

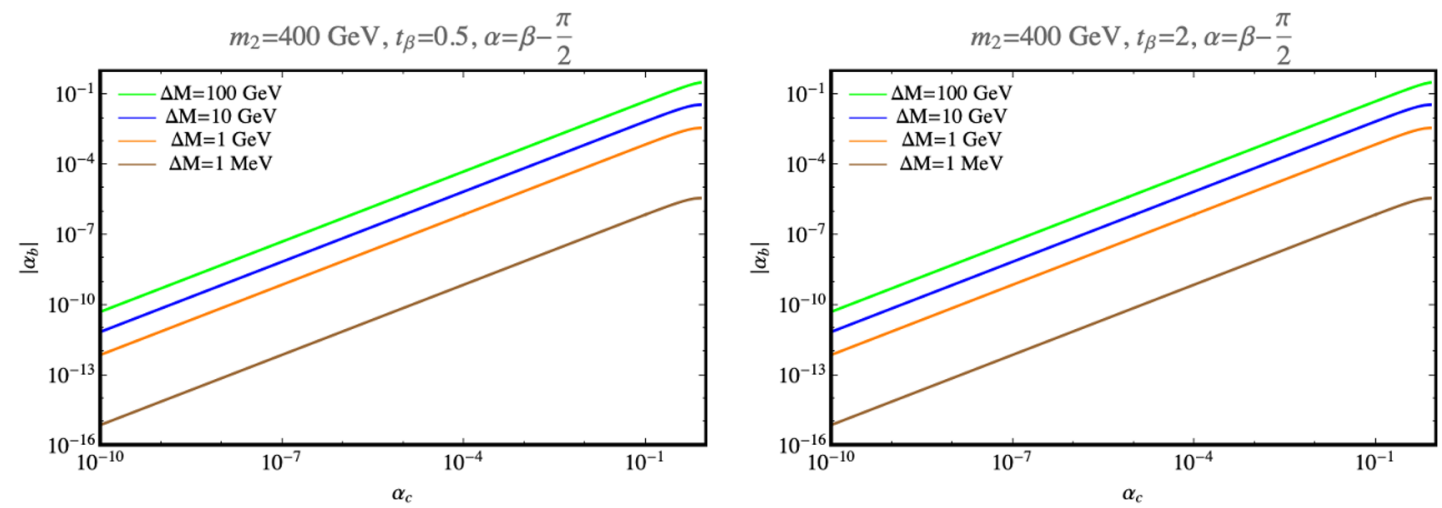

Figure 1. The values of $\left|\alpha_{b}\right|$ versus the $\alpha_{c}$ with various inputs of $\Delta M=m_{3}-m_{2}=(100 \mathrm{GeV}$, $10 \mathrm{GeV}, 1 \mathrm{GeV}, 1 \mathrm{MeV}$ ), with $t_{\beta}=0.5$ (left) and $t_{\beta}=2$ (right), and $\alpha=\beta-\frac{\pi}{2}$.

integer. Without loss of generality, we shall take $n=0$ for our later discussions. In order to simplify the calculation, we make a rephase of $\Phi_{2} \rightarrow e^{-i \delta_{1}} \Phi_{2}$ to eliminate the phases in the coefficients, so that $\operatorname{Im} m_{12}^{2}=\operatorname{Im} \lambda_{5}=0$. The minimization conditions for the SCPV scenario are then expressed as

$$
\begin{aligned}
m_{11}^{2} & =-\frac{1}{2} \lambda_{1} v^{2} c_{\beta}^{2}-\frac{1}{2}\left(\lambda_{3}+\lambda_{4}-\operatorname{Re} \lambda_{5}\right) v^{2} s_{\beta}^{2}, \\
m_{22}^{2} & =-\frac{1}{2} \lambda_{2} v^{2} s_{\beta}^{2}-\frac{1}{2}\left(\lambda_{3}+\lambda_{4}-\operatorname{Re} \lambda_{5}\right) v^{2} c_{\beta}^{2}, \\
\operatorname{Re}_{12}^{2} & =\operatorname{Re} \lambda_{5} v^{2} s_{\beta} c_{\beta} c_{\theta} .
\end{aligned}
$$

Two degenerate solutions of

$$
\theta= \pm \cos ^{-1}\left(\frac{\operatorname{Re} m_{12}^{2}}{\operatorname{Re} \lambda_{5} v^{2} s_{\beta} c_{\beta}}\right)
$$

with two opposite signs lead to the domain wall solutions. Note that this expression of $\theta$ is not our solution in the physical basis. With the minimization conditions of the SCPV scenario in eqs. (2.7), the $2 \mathrm{HDM}$ potential can be expressed as

$$
\begin{aligned}
V & =V_{\mathrm{CPC}}+V_{\mathrm{SCPV}} \\
V_{\mathrm{CPC}} & =\frac{1}{2}\left(m_{11}^{2} c_{\beta}^{2}+m_{22}^{2} s_{\beta}^{2}\right) v^{2}+\left[\frac{1}{8} \lambda_{1} c_{\beta}^{4}+\frac{1}{8} \lambda_{2} s_{\beta}^{4}+\frac{1}{4}\left(\lambda_{3}+\lambda_{4}\right) s_{\beta}^{2} c_{\beta}^{2}\right] v^{4}, \\
V_{\mathrm{SCPV}} & =-\operatorname{Re} m_{12}^{2} c_{\theta} s_{\beta} c_{\beta} v^{2}+\frac{1}{4} \operatorname{Re} \lambda_{5} c_{2 \theta} s_{\beta}^{2} c_{\beta}^{2} v^{4}=-\frac{1}{4} \operatorname{Re} \lambda_{5}\left(1+2 c_{\theta}^{2}\right) s_{\beta}^{2} c_{\beta}^{2} v^{4} .
\end{aligned}
$$

This is consistent with what was obtained in ref. [11]. The parameters in the Higgs potential (2.9) will be used for estimating the domain wall tension and the bound to the collapses later. They will be obtained by using the inputs from the physical basis, and the readers should refer to section A.1 for details.

In both the SCPV and the ECPV scenarios, the CPV mixing angles of $\alpha_{b}$ and $\alpha_{c}$ are related to each other according to eq. (A.9). Without loss of generality, we take $\alpha_{c}$ 

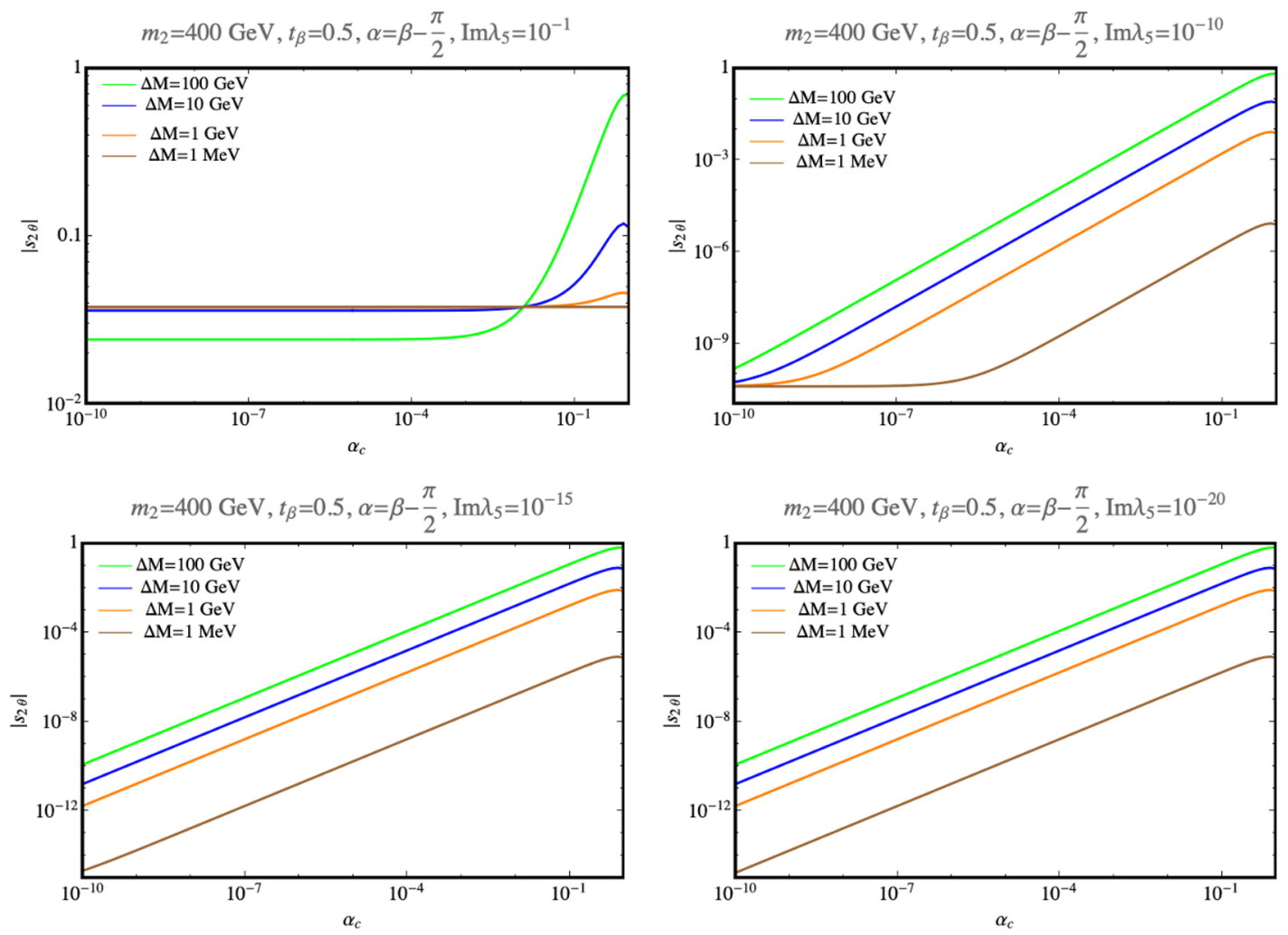

Figure 2. The values of $\left|s_{2 \theta}\right|$ versus the $\alpha_{c}$ with various inputs of $\Delta M=m_{3}-m_{2}=(100 \mathrm{GeV}$, $10 \mathrm{GeV}, 1 \mathrm{GeV}, 1 \mathrm{MeV}$ ), with $\tan \beta=0.5$ and $\alpha=\beta-\frac{\pi}{2}$. Four different values of explicity CPV parameters of $\operatorname{Im} \lambda_{5}=10^{-1}$ (upper left), $\operatorname{Im} \lambda_{5}=10^{-10}$ (upper right), $\operatorname{Im} \lambda_{5}=10^{-15}$ (lower left), and $\operatorname{Im} \lambda_{5}=10^{-20}$ (lower right) are taken.

as the input parameter. In figure 1 , we display the values of $\left|\alpha_{b}\right|$ versus the input CPV mixing angle of $\alpha_{c} \in\left(10^{-10}, 1\right)$ with various mass splittings of $\Delta M=m_{3}-m_{2}$ between two heavy neutral Higgs bosons. The decreasing values of $\alpha_{c}$ also lead to smaller values of $\left|\alpha_{b}\right|$. Also, the sizes of the CPV mixing angles are suppressed with very degenerate mass splitting of $\Delta M$, as we vary $\Delta M$ from $100 \mathrm{GeV}$ to $1 \mathrm{MeV}$ in the plots. This pattern can be found straightforwardly from eq. (A.11) as well. Therefore, the joint effects of smaller input parameter $\alpha_{c}$ and the smaller mass splitting of $\Delta M$ can lead to suppressions to the eEDM. Note that the relations of $\alpha_{b}$ versus $\alpha_{c}$ as given in eq. (A.9) and figure 1 are independent of the quartic scalar self coupling terms in the $2 \mathrm{HDM}$ potential, thus they hold for both the SCPV and the ECPV scenarios. Since the $\alpha_{b}$ dependences on $\alpha_{c}$ and the mass splitting $\Delta M$ are close between the $t_{\beta}=0.5$ and $t_{\beta}=2$ cases, we shall always use the $t_{\beta}=0.5$ as our benchmark throughout our discussions below.

\subsection{The 2HDM potential with the ECPV}

We shall solve for the relative CPV phase of $\theta$ in the ECPV scenario. Different from the SCPV scenario, the input of $\operatorname{Im} \lambda_{5}$ also plays a role. The related details are presented in section A.2. In figure 2, we depicted $\left|s_{2 \theta}\right|$ versus the CPV mixing angle of $\alpha_{c} \in\left(10^{-10}, 1\right)$ 
with various inputs of $\operatorname{Im} \lambda_{5}$. Indeed, for relatively sizeable values of $\operatorname{Im} \lambda_{5}=10^{-1}$ and $\operatorname{Im} \lambda_{5}=10^{-10}$, the solutions of $\left|s_{2 \theta}\right|$ are plateaued when $\alpha_{c}$ drops to certain threshold. Particularly for the $\operatorname{Im} \lambda_{5}=10^{-1}$ and $\Delta M=1 \mathrm{MeV}$, the values of $s_{2 \theta}$ is basically invariant with the varying $\alpha_{c}$. When the ECPV parameters are suppressed to $\operatorname{Im} \lambda_{5}=10^{-15}$ and even $\operatorname{Im} \lambda_{5}=10^{-20}$, one finds that values of $\left|s_{2 \theta}\right|$ are (almost) always decreasing with respect to the $\alpha_{c}$ inputs in the ranges of our consideration. Together, they justify our approximations in eqs. (A.21) and (A.22).

\subsection{The unitarity and stability constraints}

It is well-known that the perturbative unitarity and stability constraints to the Higgs potential should be imposed. The unitarity bounds of the $2 \mathrm{HDM}$ were previously studied in refs. $[55,56]$. The perturbative unitarity constraint means that the theory cannot be strongly coupled. In practice, the necessary and sufficient condition of the tree-level unitarity bounds can be obtained by evaluating the eigenvalues of the $S$-matrices for the scattering processes of the scalar fields in the 2HDM [55, 56]. Due to the Nambu-Goldstone theorem, the $S$-matrices can be expressed in terms of $2 \mathrm{HDM}$ quartic scalar self couplings $\lambda_{i}$. The $S$-wave amplitude matrices are due to fourteen neutral, eight singly-charged, and three doubly-charged scalar channels in the 2HDM. They read

$$
\begin{array}{rllll}
\text { neutral } a_{0}^{0}: & \left|H_{i}^{+} H_{i}^{-}\right\rangle, & \left|H_{1}^{ \pm} H_{2}^{\mp}\right\rangle, & \frac{1}{\sqrt{2}}\left|A_{i}^{0} A_{i}^{0}\right\rangle, & \frac{1}{\sqrt{2}}\left|H_{i}^{0} H_{i}^{0}\right\rangle, \\
& \left|H_{i}^{0} A_{i}^{0}\right\rangle, & \left|A_{1}^{0} A_{2}^{0}\right\rangle, & \left|H_{1}^{0} H_{2}^{0}\right\rangle, & \\
& \left|H_{1}^{0} A_{2}^{0}\right\rangle, & \left|H_{2}^{0} A_{1}^{0}\right\rangle, & & \\
\text { singly-charged } a_{0}^{+}: & \left|H_{i}^{+} A_{i}^{0}\right\rangle, & \left|H_{i}^{+} H_{i}^{0}\right\rangle, & & \\
& \left|H_{1}^{+} A_{2}^{0}\right\rangle, & \left|H_{2}^{+} A_{1}^{0}\right\rangle, & \left|H_{1}^{+} H_{2}^{0}\right\rangle, & \left|H_{2}^{+} H_{1}^{0}\right\rangle, \\
\text { doubly-charged } a_{0}^{++}: & \frac{1}{\sqrt{2}}\left|H_{i}^{ \pm} H_{i}^{ \pm}\right\rangle, & \left|H_{1}^{ \pm} H_{2}^{ \pm}\right\rangle . &
\end{array}
$$

The $S$-wave amplitude matrices for three different channels are expressed as

$$
\begin{aligned}
a_{0}^{0} & =\frac{1}{16 \pi} \operatorname{diag}\left(X_{4 \times 4}, Y_{4 \times 4}, Z_{3 \times 3}, Z_{3 \times 3}\right), \\
a_{0}^{+} & =\frac{1}{16 \pi} \operatorname{diag}\left(Y_{4 \times 4}, Z_{3 \times 3}, \lambda_{3}-\lambda_{4}\right), \\
a_{0}^{++} & =\frac{1}{16 \pi} Z_{3 \times 3} .
\end{aligned}
$$

The expressions for the submatrices of $\left(X_{4 \times 4}, Y_{4 \times 4}, Z_{3 \times 3}\right)$ are given as follows

$$
X_{4 \times 4}=\left(\begin{array}{cccc}
3 \lambda_{1} & 2 \lambda_{3}+\lambda_{4} & 0 & 0 \\
2 \lambda_{3}+\lambda_{4} & 3 \lambda_{2} & 0 & 0 \\
0 & 0 & \lambda_{3}+2 \lambda_{4}+3 \operatorname{Re} \lambda_{5} & 3 \operatorname{Im} \lambda_{5} \\
0 & 0 & 3 \operatorname{Im} \lambda_{5} & \lambda_{3}+2 \lambda_{4}-3 \operatorname{Re} \lambda_{5}
\end{array}\right)
$$




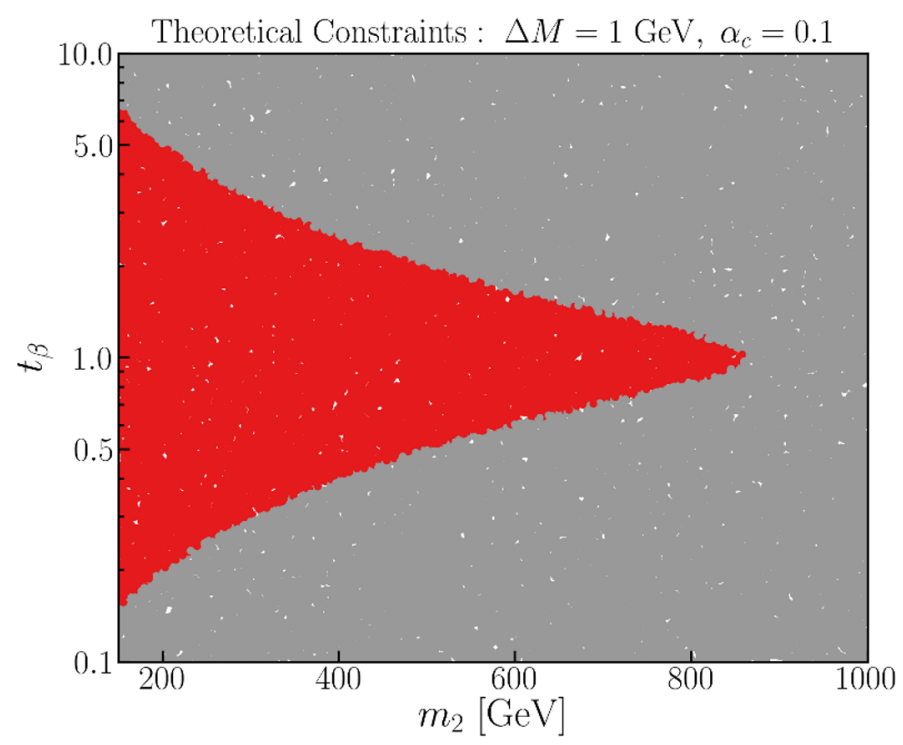

Figure 3. The theoretical constraints in the $\left(m_{2}, t_{\beta}\right)$ plane with $\Delta M=1 \mathrm{GeV}, \alpha_{c}=0.1$, where the red (grey) regions are theoretically allowed (disfavored).

$$
\begin{aligned}
Y_{4 \times 4} & =\left(\begin{array}{cccc}
\lambda_{1} & \lambda_{4} & 0 & 0 \\
\lambda_{4} & \lambda_{2} & 0 & 0 \\
0 & 0 & \lambda_{3}+\operatorname{Re} \lambda_{5} & \operatorname{Im} \lambda_{5} \\
0 & 0 & \operatorname{Im} \lambda_{5} & \lambda_{3}-\operatorname{Re} \lambda_{5}
\end{array}\right), \\
Z_{3 \times 3} & =\left(\begin{array}{ccc}
\lambda_{1} & \operatorname{Re} \lambda_{5}+i \operatorname{Im} \lambda_{5} & 0 \\
\operatorname{Re} \lambda_{5}-i \operatorname{Im} \lambda_{5} & \lambda_{2} & 0 \\
0 & 0 & \lambda_{3}+\lambda_{4}
\end{array}\right) .
\end{aligned}
$$

The unitarity requires $\left|a_{0}^{i}\right| \leq 1$.

The stability constraints require a positive 2HDM potential for large values of Higgs fields along all field space directions. Collectively, they read

$$
\lambda_{1,2}>0, \quad \lambda_{3}>-\sqrt{\lambda_{1} \lambda_{2}}, \quad \lambda_{3}+\lambda_{4}-\left|\lambda_{5}\right|>-\sqrt{\lambda_{1} \lambda_{2}} .
$$

In figure 3 , we present the theoretical constraints in the $\left(m_{2}, t_{\beta}\right)$ plane, with other parameters fixed to be $\Delta M=1 \mathrm{GeV}$ and $\alpha_{c}=0.1$. The heavy neutral Higgs boson masses are found to be bounded from above, and large $t_{\beta} \gtrsim 5.0$ or small $t_{\beta} \lesssim 0.2$ are disfavored.

\section{Domain walls in the CPV 2HDM}

In this section, we study the domain wall solutions in the $2 \mathrm{HDM}$ with the SCPV vacuum solution. ${ }^{2}$ Such solutions arise from the CP transformations of $\Phi_{j} \rightarrow \Phi_{j}^{*}$ in the $2 \mathrm{HDM}$.

\subsection{The domain wall solutions}

Under the discrete CP transformations of two Higgs doublets

$$
\Phi_{1} \rightarrow \Phi_{1}^{*}, \quad \Phi_{2} \rightarrow \Phi_{2}^{*},
$$

\footnotetext{
${ }^{2}$ This was dubbed as the CP1 domain wall in the ref. [52].
} 

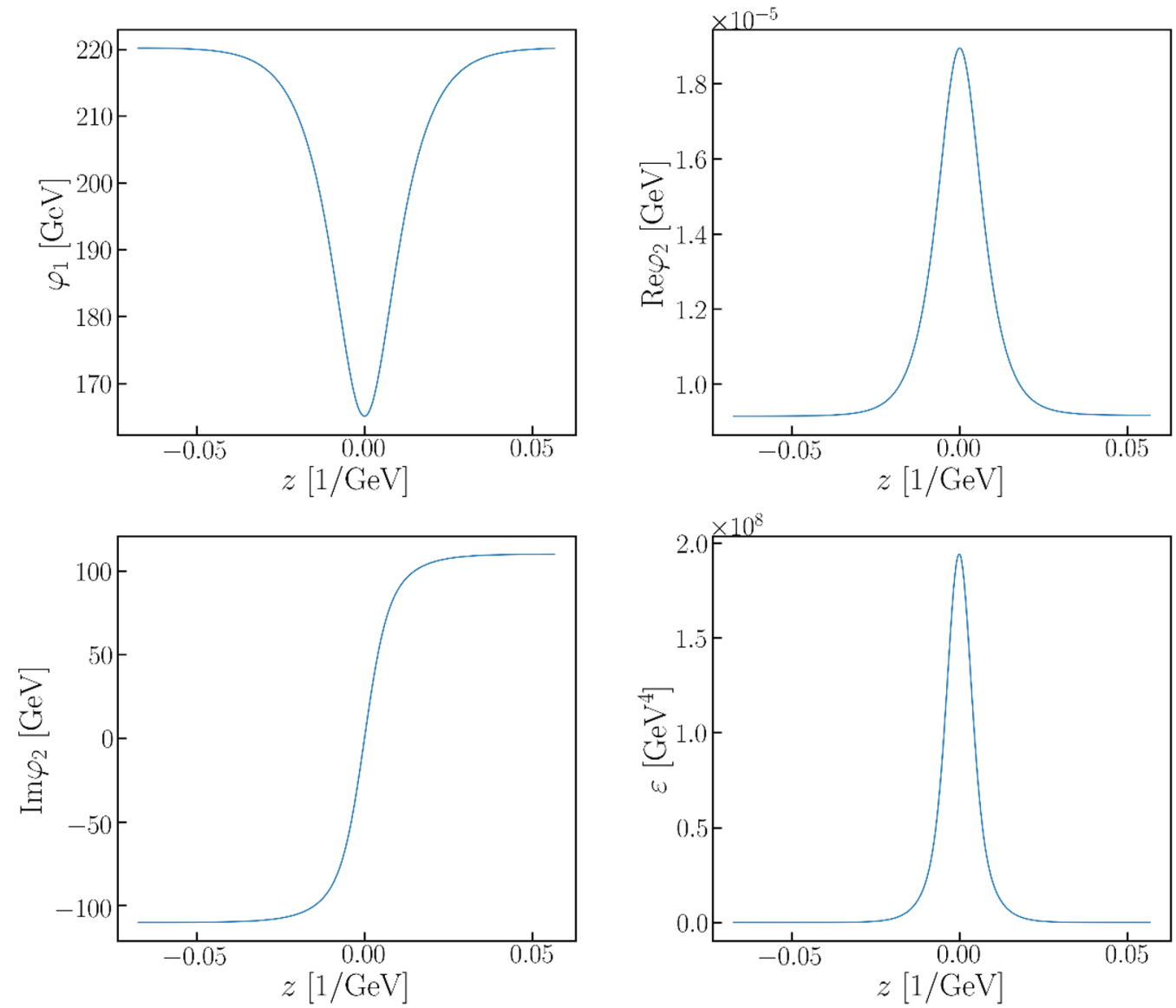

Figure 4. The profiles of the background fields and energy density across the domain wall for $m_{2}=400 \mathrm{GeV}, \Delta M=1 \mathrm{GeV}, t_{\beta}=0.5, \alpha=\beta-\pi / 2, \alpha_{c}=10^{-5}$.

one has $\Theta \rightarrow-\Theta$ for the background fields. This means the CP transformation to two Higgs doublets is equivalent to a $\mathbb{Z}_{2}$ transformation to their relative phase. Correspondingly, the CPC part of eq. (2.1) is invariant, while the CPV terms in eq. (2.1) is manifestly odd. The vacuum manifold and the corresponding nontrivial homotopy group [52, 57] is

$$
\mathcal{M}^{\mathrm{SCPV}} \simeq \mathbb{Z}_{2} \otimes S^{3}, \quad \Pi_{0}\left(\mathcal{M}^{\mathrm{SCPV}}\right) \neq \mathbf{1},
$$

where one uses the fact that the CP symmetry is homeomorphic to the $\mathbb{Z}_{2}$ symmetry, and the vacuum manifold of $\mathrm{SU}(2)_{\mathrm{L}} \times \mathrm{U}(1)_{\mathrm{Y}} / \mathrm{U}(1)_{\mathrm{em}}$ is homeomorphic to $S^{3}$. Therefore, the SCPV part of the $2 \mathrm{HDM}$ potential leads to a domain wall solution.

The domain wall solution is obtained in the 'Euclidean basis' of

$$
\vec{\phi} \equiv\left(\varphi_{1}, \operatorname{Re} \varphi_{2}, \operatorname{Im} \varphi_{2}\right)=\left(\varphi_{1}, \varphi_{2} c_{\Theta}, \varphi_{2} s_{\Theta}\right) .
$$

Two domains correspond to $\vec{\phi}=\left(v_{1}, c_{\theta} v_{2}, \pm s_{\theta} v_{2}\right)$. The energy density is given as follows:

$$
\begin{aligned}
\mathcal{E}_{\text {total }} & =\frac{1}{2}\left(\partial_{z} \vec{\phi}\right)^{2}+V(\vec{\phi}), \\
V(\vec{\phi}) & =V_{\mathrm{CPC}}+V_{\mathrm{SCPV}}+V_{\mathrm{ECPV}}+V_{0},
\end{aligned}
$$




$$
\begin{aligned}
V_{\mathrm{CPC}} & =\frac{1}{2} m_{11}^{2} \varphi_{1}^{2}+\frac{1}{2} m_{22}^{2} \varphi_{2}^{2}+\frac{\lambda_{1}}{8} \varphi_{1}^{4}+\frac{\lambda_{2}}{8} \varphi_{2}^{4}+\frac{1}{4}\left(\lambda_{3}+\lambda_{4}\right) \varphi_{1}^{2} \varphi_{2}^{2}, \\
V_{\mathrm{SCPV}} & =-\operatorname{Re} m_{12}^{2} \varphi_{1} \varphi_{2}+\frac{1}{4} \operatorname{Re} \lambda_{5} \varphi_{1}^{2} \varphi_{2}^{2}, \\
V_{\mathrm{ECPV}} & =-\frac{1}{4} \operatorname{Im} \lambda_{5} \varphi_{1}^{2} \varphi_{2}^{2} s_{2 \Theta} \\
V_{0} & =\frac{1}{8}\left(\lambda_{1} v_{1}^{4}+\lambda_{2} v_{2}^{4}\right)+\frac{1}{4}\left(\lambda_{3}+\lambda_{4}+\operatorname{Re} \lambda_{5} c_{2 \theta}\right) v_{1}^{2} v_{2}^{2} .
\end{aligned}
$$

where $V_{0}$ is a pure constant to make the potential of electroweak vacuum zero. In our real calculation of the domain wall profile, $V_{\mathrm{ECPV}}$ should be taken into account, as it shifts the potential as well as the local minima positions. However, when considering the case $\left|\operatorname{Im} \lambda_{5}\right| \ll 1$, we can safely ignore the ECPV part when solving the domain wall profile. The tension of the domain wall is then the integral of the total energy density:

$$
\sigma \simeq \int_{-\infty}^{\infty} d z\left[\frac{1}{2}\left(\partial_{z} \vec{\phi}\right)^{2}+V(\vec{\phi})\right]
$$

The corresponding domain wall profile is obtained by solving the equations of motion (EOM) of $\vec{\phi}$ :

$$
\frac{d^{2}}{d z^{2}} \vec{\phi}=\vec{\nabla}_{\phi} V(\vec{\phi})
$$

with the boundary conditions being

$$
\vec{\phi}(z=\mp \infty)=\left(v_{1}, v_{2} c_{\theta}, \mp v_{2} s_{\theta}\right) .
$$

The EOM is solved using the path deformation algorithm ${ }^{3}[25,58]$. In figure 4 , we display a sample of the domain wall profiles and the energy density of $\mathcal{E}(z)$. Since we expect that the relative $\mathrm{CPV}$ phase to be $\theta \rightarrow \pm \pi / 2$, thus, the imaginary component of $\varphi_{2}$ takes much larger value comparing to the real component. In our numerical estimation, we find that the domain wall tensions from eq. (3.5) are typically $\sim \mathcal{O}\left(10^{6}\right) \mathrm{GeV}^{3}$. One can quickly find this result from the energy density plot of our sample in figure 4, where the local energy density is roughly $\mathcal{E} \sim \mathcal{O}\left(10^{8}\right) \mathrm{GeV}^{4}$ and the domain wall width is around $\sim \mathcal{O}(0.01) \mathrm{GeV}^{-1}$.

\subsection{The biased term from the ECPV}

The ECPV component of the potential leads to additional contribution to the energy density as below

$$
V_{\mathrm{ECPV}}(z)=-\frac{1}{4} \operatorname{Im} \lambda_{5} \varphi_{1}^{2} \varphi_{2}^{2} s_{2 \Theta}
$$

which becomes the biased term for the SCPV domain walls. After the EWSB, the corresponding biased term reads

$$
\begin{aligned}
\Delta V & =\left|V_{\mathrm{ECPV}}(z=+\infty)-V_{\mathrm{ECPV}}(z=-\infty)\right| \\
& =\frac{1}{2}\left|\operatorname{Im} \lambda_{5} s_{2 \theta}\right| v_{1}^{2} v_{2}^{2},
\end{aligned}
$$

with the relative CPV phase of $\theta$ being solved from eq. (A.20).

\footnotetext{
${ }^{3}$ The corresponding code can be found in https://github.com/ycwu1030/BSM_Soliton.
} 


\subsection{The cosmological constraints}

The observation of the CMBR leads to the following condition to the domain wall tension

$$
\sigma \lesssim \mathcal{O}(1) \mathrm{MeV}^{3}
$$

which was known as the Zel'dovich-Kobzarev-Okun bound. Our numerical solutions found that the domain wall solutions in the SCPV case generally lead to tensions of $\sigma \sim \mathcal{O}\left(10^{6}\right) \mathrm{GeV}^{3}$, which is $\sim 10^{15}$ times above the Zel'dovich-Kobzarev-Okun bound. Therefore, the biased term from the ECPV is necessary for the domain wall collapse.

To have the large scale domain wall to form, it is known that the energy difference between two vacua should be sufficiently small:

$$
\frac{\Delta V}{\left|V_{0}\right|}<\log \frac{1-p_{c}}{p_{c}}=0.795
$$

with $\left|V_{0}\right|$ representing the height of the potential barrier between two minima in eq. (3.4f), and the critical value of $p_{c}=0.311$ predicted from the percolation theory [59].

Lower bounds can be also obtained to the energy difference. The domain wall cannot exist too long to spoil the known constraints from the BBN [23, 60, 61]. This leads to an lower bound to the energy difference

$$
\Delta V^{1 / 4} \gtrsim 5.07 \times 10^{-4} \mathrm{GeV} C_{\mathrm{ann}}^{1 / 4} \mathcal{A}^{1 / 4} \hat{\sigma}^{1 / 4}
$$

with $\mathcal{A} \sim 0.8 \pm 0.1$ [62], and $C_{\text {ann }}=2$ for the $\mathbb{Z}_{2}$ symmetry. $\hat{\sigma} \equiv \sigma /(1 \mathrm{TeV})^{3}$ denotes the dimensionless domain wall tension. Assuming that the domain wall collapse occurred during the radiation dominated era, the corresponding temperature is given by

$$
T_{\text {ann }}=3.41 \times 10^{-2} \mathrm{GeV} C_{\text {ann }}^{-1 / 2} \mathcal{A}^{-1 / 2}\left(\frac{g_{*}\left(T_{\text {ann }}\right)}{10}\right)^{-1 / 4} \hat{\sigma}^{-1 / 2} \Delta \hat{V}^{1 / 2},
$$

with $g_{*}\left(T_{\text {ann }}\right)$ counting the relativistic degrees of freedom at the annihilation temperature, and $\Delta \hat{V} \equiv \Delta V /(1 \mathrm{MeV})^{4}$.

In figure 5 , we present the size of the biased term $\Delta V$ versus the CPV mixing angle $\alpha_{c} \in\left(10^{-10}, 1\right)$, for different mass splittings of $\Delta M=(100 \mathrm{GeV}, 10 \mathrm{GeV}, 1 \mathrm{GeV}, 1 \mathrm{MeV})$ between two neutral heavy Higgs bosons. The upper bound from eq. (3.11) and lower bound from eq. (3.12) are presented in terms of dotted (in purple) and dashed (in black) lines, respectively. It turns out that the upper bound can be always satisfied with the parameter choices of $\alpha_{c}$ in our considerations. Meanwhile, the lower bounds to the domain wall collapse can become sensitive to the CPV mixing angle of $\alpha_{c}$ only when the explicit CPV parameter of $\operatorname{Im} \lambda_{5}$ is sufficiently small. This can be expected from our previous discussions about the $t_{\theta}$ dependences on the physical inputs of $\alpha_{c}$ and $\operatorname{Im} \lambda_{5}$. For the $\operatorname{Im} \lambda_{5}=10^{-10}$ case, one also finds that $\Delta V$ becomes plateaued similar to the corresponding $\left|s_{2 \theta}\right|$ plot in figure 2. On the other hand, too small $\Delta M$ and/or $\operatorname{Im} \lambda_{5}$ values are excluded by the BBN lower limit as shown in the lower panels of figure 5 . 

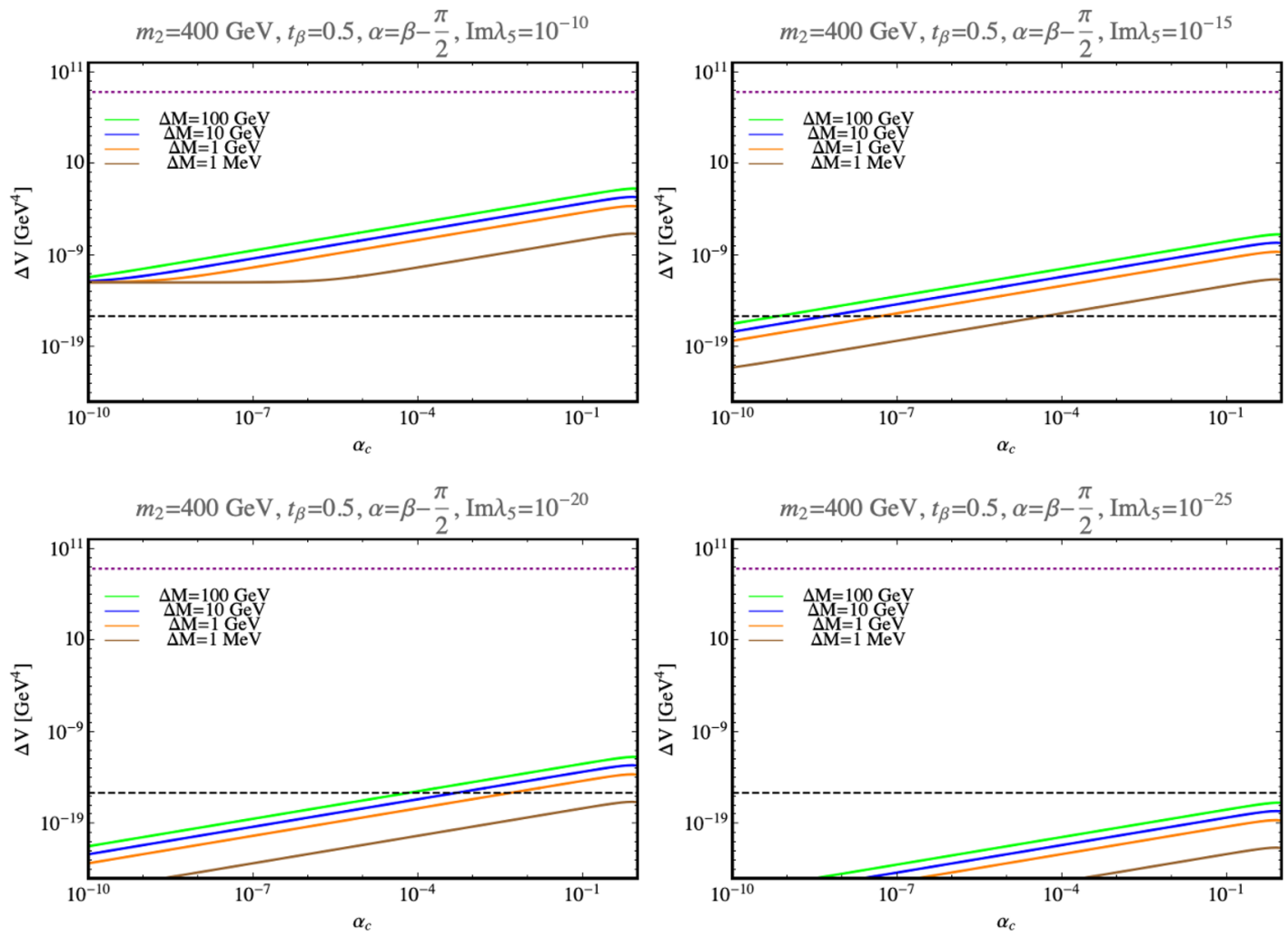

Figure 5. The $\Delta V$ versus the $\alpha_{c}$ with various inputs of: $\operatorname{Im} \lambda_{5}=10^{-10}$ (upper-left), $\operatorname{Im} \lambda_{5}=10^{-15}$ (upper-right), $\operatorname{Im} \lambda_{5}=10^{-20}$ (lower-left), and $\operatorname{Im} \lambda_{5}=10^{-25}$ (lower-right). In each plot, four different mass splittings of $\Delta M=(100 \mathrm{GeV}, 10 \mathrm{GeV}, 1 \mathrm{GeV}, 1 \mathrm{MeV})$ are presented. Other input parameters are $m_{2}=400 \mathrm{GeV}$ and $t_{\beta}=0.5$. The dotted lines represent the upper bound to the domain wall formation in eq. (3.11), and the dashed lines represent the lower bound to the domain wall collapse in eq. (3.12).

\subsection{The GW signals}

The collapsing domain walls can lead to GW signals [21-23, 62, 63], while we find that such signals arising from the CPV $2 \mathrm{HDM}$ are impossible to be probed in any of the future satellite observations. We shall briefly discuss the signal estimations below.

The peak frequency of the GWs at the annihilation time of domain walls is proportional to the annihilation temperature $T_{\mathrm{ann}}$ in eq. (3.13), and is given by

$$
\begin{aligned}
f_{\text {peak }} & \simeq 1.1 \times 10^{-7} \mathrm{~Hz}\left(\frac{g_{*}\left(T_{\text {ann }}\right)}{10}\right)^{1 / 2}\left(\frac{g_{* s}\left(T_{\text {ann }}\right)}{10}\right)^{-1 / 3}\left(\frac{T_{\text {ann }}}{1 \mathrm{GeV}}\right) \\
& \simeq 3.75 \times 10^{-9} \mathrm{~Hz}\left(\frac{g_{*}\left(T_{\text {ann }}\right)}{10}\right)^{1 / 4}\left(\frac{g_{* s}\left(T_{\text {ann }}\right)}{10}\right)^{-1 / 3} C_{\text {ann }}^{-1 / 2} \mathcal{A}^{-1 / 2} \hat{\sigma}^{-1 / 2} \Delta \hat{V}^{1 / 2} .
\end{aligned}
$$

Here, $g_{*}\left(T_{\text {ann }}\right)$ and $g_{* s}\left(T_{\text {ann }}\right)$ count the relativistic degrees of freedom contributing to the energy density and the entropy density. They are both 10.75 for $1 \mathrm{MeV} \lesssim \mathrm{T}_{\text {ann }} \lesssim 100 \mathrm{MeV}$. For GWs with peak frequencies in the range of $\mathcal{O}\left(10^{-4}\right)-\mathcal{O}\left(10^{-1}\right) \mathrm{Hz}$, they may be probed by the future satellite-based interferometers, such as the LISA [27, 28], Taiji [29], and 
Tianqin [30] programs. The GWs with very small peak frequencies of few nano $\mathrm{Hz}$ may be probed at the future radio telescope of SKA [31] and the DECIGO [32] with the latter having wider range of typical frequencies of $\sim \mathcal{O}(0.1)-\mathcal{O}(10) \mathrm{Hz}$. With the lower limit of the $\Delta V$ in eq. (3.12), there is a lower limit to the peak frequency of

$$
f_{\text {peak }} \gtrsim 0.94 \times 10^{-9} \mathrm{~Hz}\left(\frac{g_{*}\left(T_{\mathrm{ann}}\right)}{10}\right)^{1 / 4}\left(\frac{g_{* s}\left(T_{\mathrm{ann}}\right)}{10}\right)^{-1 / 3} .
$$

Thus, the peak frequencies of the GW signals are expected to be higher than order of several nano Hz.

The peak energy density spectrum of the GW is

$$
\Omega_{\mathrm{GW}}^{\mathrm{peak}} h^{2}\left(t_{0}\right)=7.2 \times 10^{-18} \tilde{\epsilon}_{\mathrm{GW}} \mathcal{A}^{2}\left(\frac{g_{* s}\left(T_{\mathrm{ann}}\right)}{10}\right)^{-4 / 3}\left(\frac{T_{\mathrm{ann}}}{10^{-2} \mathrm{GeV}}\right)^{-4} \hat{\sigma}^{2},
$$

with $\tilde{\epsilon}_{\mathrm{GW}} \simeq 0.7 \pm 0.4$ in the scaling regime [62]. By using the annihilation temperature in eq. (3.13), the peak energy density spectrum becomes

$$
\Omega_{\mathrm{GW}}^{\mathrm{peak}} h^{2}\left(t_{0}\right)=5.3 \times 10^{-20} \tilde{\epsilon}_{\mathrm{GW}} \mathcal{A}^{4} C_{\mathrm{ann}}^{2}\left(\frac{g_{* s}\left(T_{\mathrm{ann}}\right)}{10}\right)^{-4 / 3}\left(\frac{g_{*}\left(T_{\mathrm{ann}}\right)}{10}\right) \hat{\sigma}^{4} \Delta \hat{V}^{-2} .
$$

By taking the lower limit of the $\Delta V$ in eq. (3.12) into account, we find an upper limit to the peak energy density spectrum as

$$
\Omega_{\mathrm{GW}}^{\text {peak }} h^{2}\left(t_{0}\right) \lesssim 1.36 \times 10^{-17} \tilde{\epsilon}_{\mathrm{GW}} \mathcal{A}^{2}\left(\frac{g_{* s}\left(T_{\mathrm{ann}}\right)}{10}\right)^{-4 / 3}\left(\frac{g_{*}\left(T_{\mathrm{ann}}\right)}{10}\right) \hat{\sigma}^{2} .
$$

With the lower limit of the peak frequencies $\gtrsim 10^{-9} \mathrm{~Hz}$ in eq. (3.15), and the peak energy density to be less than $\sim \mathcal{O}\left(10^{-23}\right)$, by using the facts that $\sigma \sim \mathcal{O}\left(10^{6}\right) \mathrm{GeV}^{3}$ through the numerical results. The related GW signals from the domain wall collapsing are roughly $\sim \mathcal{O}\left(10^{-8}\right)$ below the search sensitivities of SKA.

\section{The EDM measurements}

Obviously, the EDM measurements provide us direct constraints to the size of the CPV. They can provide exclusive bound on the size of CPV in 2HDM together with the cosmological constraint on domain wall. In this work, we focus on the evaluations of the eEDM. We estimate the eEDM from the CPV 2HDM with the domain wall solutions, together with the ECPV parameters. The latest upper bound of the electric dipole moment of the electron from the ACME-II [34] reads

$$
\text { ACME-II : }\left|\frac{d_{e}}{e}\right| \leq 1.1 \times 10^{-29} \mathrm{~cm},
$$

and the future projected precision from the ACME-III reads ${ }^{4}$

$$
\text { ACME-III : }\left|\frac{d_{e}}{e}\right| \lesssim 1.0 \times 10^{-30} \mathrm{~cm} .
$$

\footnotetext{
${ }^{4}$ This projection can be found in http://www.electronedm.org/.
} 

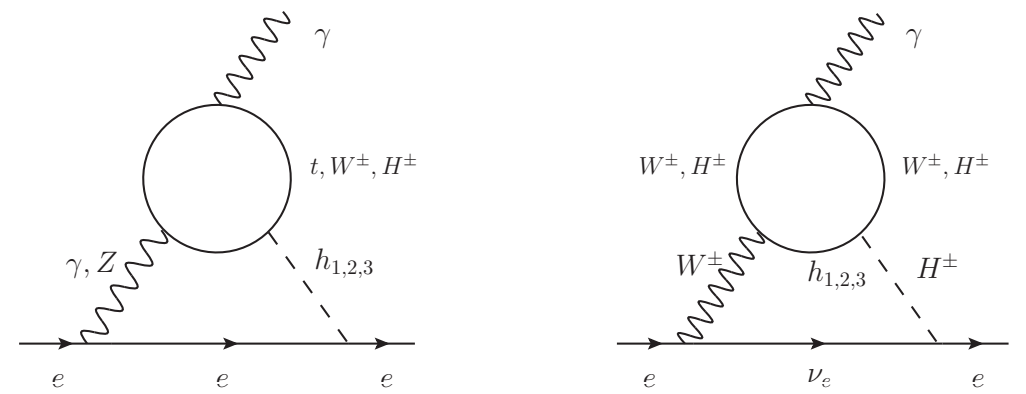

Figure 6. The two-loop Barr-Zee diagrams for the $h_{i} \gamma \gamma, h_{i} \gamma Z$, and $H^{ \pm} W^{\mp} \gamma$ operators of the eEDM contributions.

The SM estimations of the eEDM were of size $\left|d_{e} / e\right| \sim 10^{-44} \mathrm{~cm}$ from the four-loop contributions of the CKM phase, and $\left|d_{e} / e\right| \sim 10^{-38}-10^{-39} \mathrm{~cm}$ by considering the CP-odd electron-nucleon interaction [64-67].

The dominant contributions to the eEDMs come from the two-loop Barr-Zee diagrams [68] in the CPV 2HDM. There are three types of dimension-five operators involved: (i) the $h_{i} \gamma \gamma$ operator, (ii) the $h_{i} \gamma Z$ operator, and (iii) the $H^{ \pm} W^{\mp} \gamma$ operator. Expressed in terms of the Wilson coefficient of these operators, we summarize the total contributions as follows

$$
\begin{aligned}
\delta_{e}= & \left(\delta_{e}\right)_{t}^{h_{i} \gamma \gamma}+\left(\delta_{e}\right)_{W}^{h_{i} \gamma \gamma}+\left(\delta_{e}\right)_{H^{ \pm}}^{h_{i} \gamma \gamma} \\
& +\left(\delta_{e}\right)_{t}^{h_{i} \gamma Z}+\left(\delta_{e}\right)_{W}^{h_{i} \gamma Z}+\left(\delta_{e}\right)_{H^{ \pm}}^{h_{i} \gamma}+\left(\delta_{e}\right)_{h_{i}}^{H^{ \pm} W^{\mp} \gamma}
\end{aligned}
$$

and the corresponding diagrams are depicted in figure 6. Explicit expressions of these Wilson coefficients can be found in the appendices of refs. [38, 44]. By combining the total contributions in eq. (4.3), the eEDM is obtained by

$$
\frac{d_{e}}{e}=\frac{2 m_{e}}{v^{2}} \delta_{e} .
$$

The Wilson coefficients are generally related to the normalized scalar or pseudoscalar Yukawa couplings as

$$
\left(\delta_{e}\right)_{t} \propto c_{\ell, i} \tilde{c}_{u, i} / \tilde{c}_{\ell, i} c_{t, i}, \quad\left(\delta_{e}\right)_{W} \propto a_{i} \tilde{c}_{\ell, i}
$$

The pseudoscalar couplings are all proportional to the CPV mixing angles as $\tilde{c}_{f, i} \propto \alpha_{c}$, which can be found in table 3 and the relation of eq. (A.11). One can expect that future improvements of the eEDM precisions by an order of magnitude will further constrain the CPV mixing angle by an order of magnitude.

We display the eEDM predictions of $\left|d_{e} / e\right|$ versus the CPV mixing angle of $\alpha_{c}$ in figure 7. The latest upper bound to the eEDM from the ACME-II from eq. (4.1) and the future projected upper bound from the ACME-III in eq. (4.2) are displayed in horizontal dashed lines. The evaluations of the eEDM depend on the mixing angles of $\left(\alpha, \beta, \alpha_{b}, \alpha_{c}\right)$. By using the constraint for masses and mixing angles in eq. (A.9), the mass splitting of $\Delta M$ can also play a role in the size of the eEDM predictions. With a fixed input of $\alpha_{c}$, 

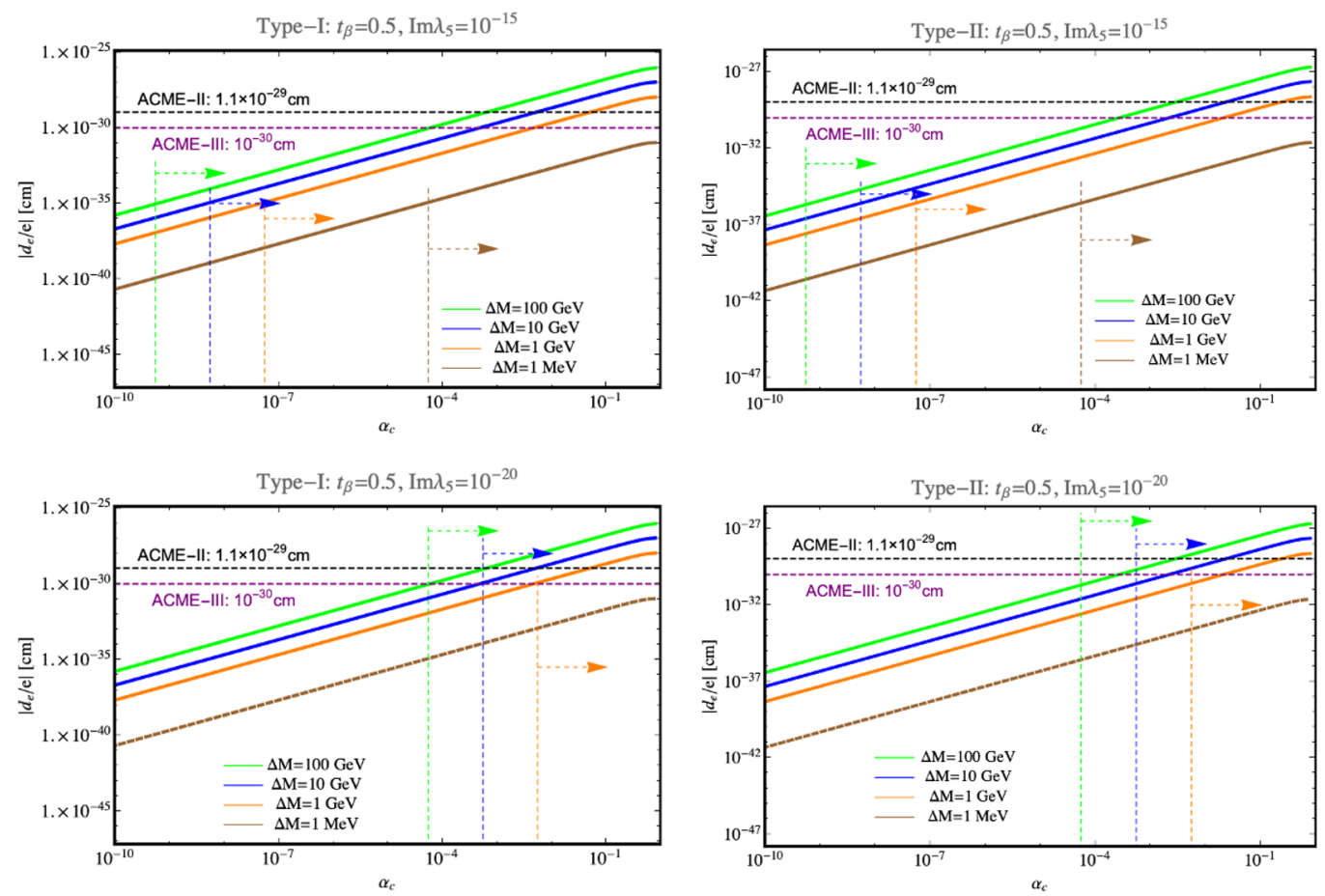

Figure 7. The eEDM predictions versus the CPV mixing angle $\alpha_{c}$ in the range of $\alpha_{c} \in$ $\left(10^{-10}, 1\right)$. The lower limits to $\alpha_{c}$ are shown in arrows for the $\operatorname{Im} \lambda_{5}=10^{-15}$ (upper panels) and $\operatorname{Im} \lambda_{5}=10^{-20}$ (lower panels) cases from the BBN bound to the biased term, as obtained from eq. (3.12). For each case, we display the eEDM predictions for different mass splittings of $\Delta M=(100 \mathrm{GeV}, 10 \mathrm{GeV}, 1 \mathrm{GeV}, 1 \mathrm{MeV})$.

one generally has a suppressed value of $\alpha_{b}$ with smaller inputs of $\Delta M$, as was displayed in figure 1. Consequently, the eEDM predictions will be suppressed as well. This was previously discussed in ref. [41]. The evaluations of the eEDMs are independent of the size of ECPV parameter $\operatorname{Im} \lambda_{5}$, as one can visualize between two upper panels and two lower panels in figure 7. Meanwhile, different inputs of $\operatorname{Im} \lambda_{5}$ lead to different cosmological constraints to the $\alpha_{c}$ through eq. (3.12). Explicitly, these lower bounds to $\alpha_{c}$ are denoted by dashed vertical lines with arrows in each plot. For the $\operatorname{Im} \lambda_{5}=10^{-15}$ case, there are lower limits to the CPV mixing angle of $\alpha_{c}$ for all four $\Delta M$ inputs. However, when such lower limits of $\alpha_{c}$ are saturated, the corresponding eEDM predictions are $\left|d_{e} / e\right| \sim \mathcal{O}\left(10^{-35}\right) \mathrm{cm}$, which is another five orders of magnitude below the future precision aimed at the ACME-III. When one further reduces the ECPV parameter to $\operatorname{Im} \lambda_{5}=10^{-20}$, the constraint of eq. (3.12) has already ruled out the situation with small mass splitting of $\Delta M=1 \mathrm{MeV}$. This can be also observed in the lower-left panel of figure 5. Thus, we denote the $\Delta M=1 \mathrm{MeV}$ with $\operatorname{Im} \lambda_{5}=10^{-20}$ cases by dashed lines, indicating that their exclusion from the BBN constraint. We also checked that for the $\operatorname{Im} \lambda_{5}=10^{-20}$ case, the lower limits of $\alpha_{c}$ from eq. (3.12) lead to the eEDM predictions of $\left|d_{e} / e\right| \sim \mathcal{O}\left(10^{-30}\right) \mathrm{cm}$. Therefore, the situation with very tiny ECPV parameter of $\operatorname{Im} \lambda_{5}=10^{-20}$ is expected to be confirmed or excluded with the joint BBN constraints and the improved experimental precision of the eEDM from the future ACME-III. 

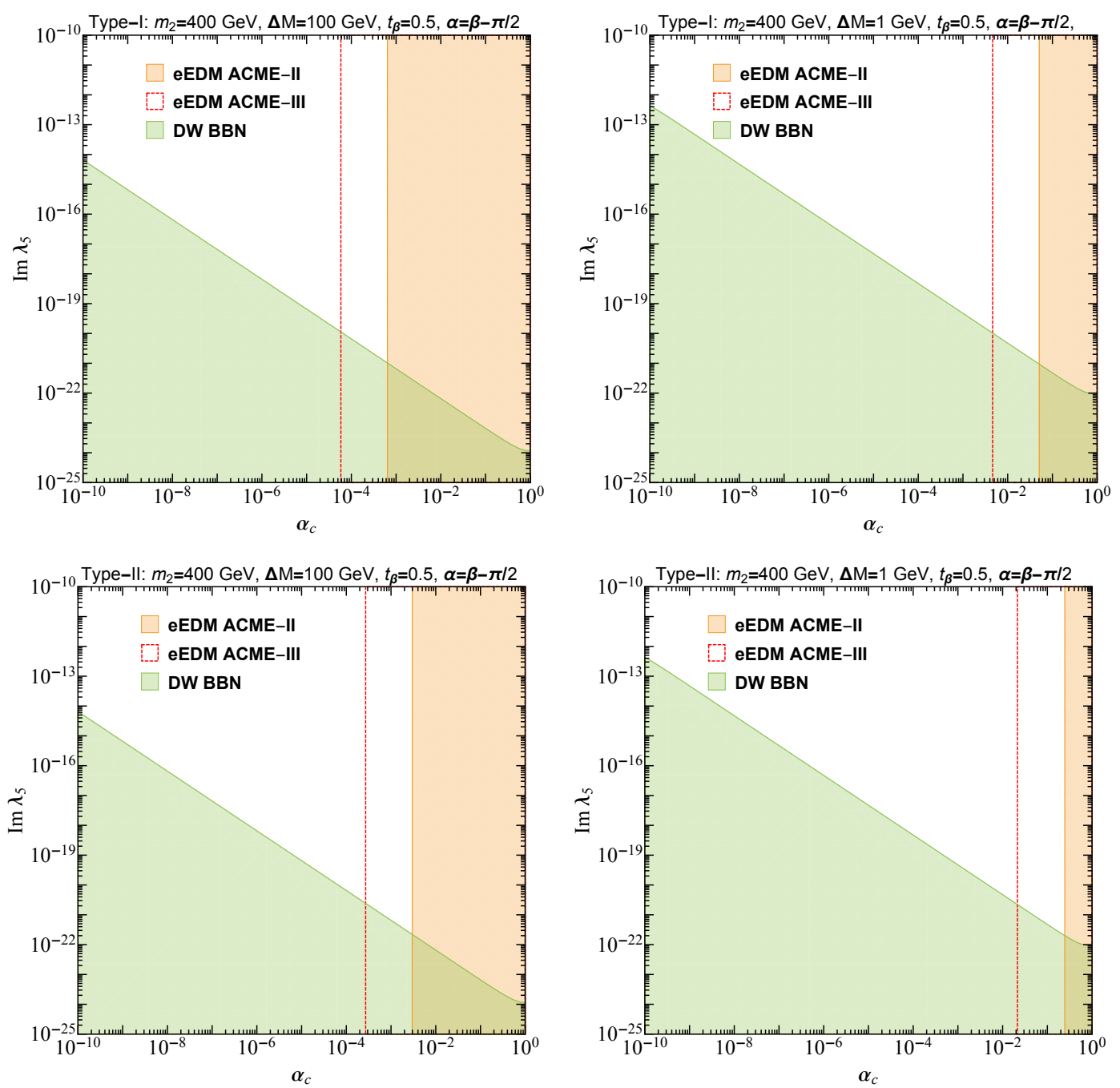

Figure 8. The joint BBN and eEDM constraints in the $\left(\alpha_{c}, \operatorname{Im} \lambda_{5}\right)$ plane. The colored regions are excluded by corresponding constraints. The dashed lines correspond to the future improvements to the eEDM measurements from the ACME-III.

In figure 8, we further present the joint BBN constraint from eq. (3.12) and the eEDM measurements from the current ACME-II limits (4.1) (orange shaded regions) and the future ACME-III projections (4.2) (vertical dashed lines). Two different mass splittings of $\Delta M=100 \mathrm{GeV}$ (left panels) and $\Delta M=1 \mathrm{GeV}$ (right panels) are displayed. With the future improvements of the eEDM precision by an order of magnitude, the corresponding constraints to the CPV mixing of $\alpha_{c}$ will be improved by an order of magnitude accordingly. For a relatively large mass splittings of $\Delta M=100 \mathrm{GeV}$, the upper limits to $\alpha_{c}$ from the future eEDM measurements can be as small as $\sim \mathcal{O}\left(10^{-4}\right)$. While for a suppressed mass splittings of $\Delta M=1 \mathrm{GeV}$, the upper limits to $\alpha_{c}$ become $\sim \mathcal{O}\left(10^{-2}\right)$. This is due to the relation between $\left|\alpha_{b}\right|$ versus $\alpha_{c}$ as given in eq. (A.11) and figure 1.

Furthermore, with the BBN constraints to the biased domain wall term, the sizes of the ECPV parameter $\operatorname{Im} \lambda_{5}$ are constrained with various inputs of the physical CPV mixing 
angle $\alpha_{c}$. Such constraints are becoming more stringent with smaller inputs of $\alpha_{c}$. For a fixed value of $\operatorname{Im} \lambda_{5}$, a lower limit to $\alpha_{c}$ is given in the light green region. We find that $\operatorname{Im} \lambda_{5}$ should be $\gtrsim \mathcal{O}\left(10^{-14}\right)\left(\gtrsim \mathcal{O}\left(10^{-24}\right)\right.$ with the $\mathrm{CPV}$ mixing angles of $\alpha_{c} \sim \mathcal{O}\left(10^{-10}\right)$ $\left(\alpha_{c} \sim \mathcal{O}(1)\right)$. Joined with the current upper limits to the eEDM, we find that the incredibly tiny ECPV parameter $\operatorname{Im} \lambda_{5}$ of $\sim \mathcal{O}\left(10^{-24}\right)-\mathcal{O}\left(10^{-21}\right)$ (Type-I) or $\sim \mathcal{O}\left(10^{-24}\right)-\mathcal{O}\left(10^{-22}\right)$ (Type-II) have been ruled out in the collapsing domain wall scenario. Another order of magnitude from the future improvements to the eEDM will correspondingly constrain the tiny ECPV parameter in the extended ranges of $\sim \mathcal{O}\left(10^{-24}\right)-\mathcal{O}\left(10^{-20}\right)$ (Type-I) or $\sim \mathcal{O}\left(10^{-24}\right)-\mathcal{O}\left(10^{-21}\right)$ (Type-II)

\section{Conclusion and discussion}

In this work, we focus on the vacuum with the SCPV in the 2HDM, which can lead to a domain wall structure. Through our numerical studies, such domain walls typically lead to incredibly large tensions of $\mathcal{O}\left(10^{6}\right) \mathrm{GeV}^{3}$, which is well above the Zel'dovich-KobzarevOkun bound. Therefore, the complex parameters in the 2HDM potential are necessary in playing the role as the biased terms to collapse these domain walls. With reasonable sizes of the biased terms, such domain walls could have been formed in the early Universe. In order not to spoil the BBN constraints, we find the direct constraints to the sizes of the ECPV terms, for which we choose to be $\operatorname{Im} \lambda_{5}$, to be $\gtrsim \mathcal{O}\left(10^{-14}-10^{-12}\right)\left(\gtrsim \mathcal{O}\left(10^{-24}-10^{-22}\right)\right.$ with the CPV mixing angles of $\alpha_{c} \sim \mathcal{O}\left(10^{-10}\right)\left(\alpha_{c} \sim \mathcal{O}(1)\right)$. Although the related domain wall collapse does not lead to sufficiently strong signals for the future GW probes at the SKA, we find that the eEDM measurements will play a role to probe the deep echos from this process. The future projection of the eEDM measurements from the ACME-III can set upper limits to the CPV mixing angle of $\alpha_{c} \lesssim \mathcal{O}\left(10^{-2}\right)-\mathcal{O}\left(10^{-4}\right)$, which depends on the types of the Yuakwa couplings and mass spliting of two neutral heavy Higgs bosons. For the first time, we find the current and future eEDM measurements have excluded or can be used to probe the very tiny regions of the ECPV parameter of $\operatorname{Im} \lambda_{5} \sim \mathcal{O}\left(10^{-24}\right)-\mathcal{O}\left(10^{-20}\right)$ (Type-I) or $\operatorname{Im} \lambda_{5} \sim \mathcal{O}\left(10^{-24}\right)-\mathcal{O}\left(10^{-21}\right)$ (Type-II). In other words, we find that the eEDM measurements can look deep into the possible domain wall collapses in the early Universe. This is different from the discussions in the context of SUSY where the improved eEDM measurements were thought to set lower limit to the SUSY-breaking scale. In the context of the domain wall collapsing triggered by the complex parameters in the 2HDM potential, the future improvements of the eEDM measurements are found to further set up upper limits to the CPV mixing angles and the sizes of the ECPV term.

Some future efforts can be envisioned from this work.

1. The 2HDM, along with other new physics models, are known to produce other topological defects besides of the domain wall solutions from the CP symmetry, such as vortices and monopoles $[52,57,69-73]$. The solutions to these structure can lead to GW signals as well. Therefore, it will be useful to probe the complete spectrum of the GWs for given new physics model.

2. We did not consider the electroweak phase transition and the possibility of achieving the BAU within this frame. It was known that various topological defects, such as 
monopoles, may play a role of producing the baryon number violation process. ${ }^{5}$ There have been some recent progresses [77-79] along this direction. It is therefore to ask if a successful BAU can be achieved when the new physics models have non-trivial topological solutions.

3. From the perspective of the EDM measurements, it will be also useful to perform the estimations to the atomic EDMs and find the future experimental search projections as well.

\section{Acknowledgments}

We would like to thank Yuan Sun, Tian Xia, Ke-Pan Xie and Yue Zhang for very useful discussions and communication. The work of NC is partially supported by the National Natural Science Foundation of China (under Grant No. 11575176). TL is supported by the National Natural Science Foundation of China (Grant No. 11975129) and "the Fundamental Research Funds for the Central Universities", Nankai University (Grant No. 63196013). The work of NC and TL is also supported in part by the National Natural Science Foundation of China (Grant No. 12035008). YW is supported by the Natural Sciences and Engineering Research Council of Canada (NSERC).

\section{A The mass spectrum and self couplings with the SCPV and the ECPV}

In this appendix, we provide explicit relations between the $2 \mathrm{HDM}$ parameters in the generical basis and the physical basis. Many details of deriving the relative CPV phase $\theta$ in the SCPV and the ECPV scenarios are also presented.

\section{A.1 The mass spectrum and self couplings with the SCPV}

For the SCPV scenario, we have the charged Higgs boson mass squared matrix of

$$
\mathcal{M}_{ \pm}^{2} / v^{2}=-\frac{1}{2}\left(\lambda_{4}-\operatorname{Re} \lambda_{5}\right)\left(\begin{array}{cc}
s_{\beta}^{2} & -s_{\beta} c_{\beta} e^{i \theta} \\
-s_{\beta} c_{\beta} e^{-i \theta} & c_{\beta}^{2}
\end{array}\right),
$$

and the corresponding eigenvalues are

$$
m_{ \pm}^{2}=-\frac{1}{2}\left(\lambda_{4}-\operatorname{Re} \lambda_{5}\right) v^{2}
$$

The gauge eigenstates of $\left(H_{1}^{ \pm}, H_{2}^{ \pm}\right)$are transformed into mass eigenstates of charged Nambu-Goldstone bosons $G^{ \pm}$and Higgs bosons $H^{ \pm}$by

$$
\left(\begin{array}{c}
H_{1}^{ \pm} \\
H_{2}^{ \pm}
\end{array}\right)=\left(\begin{array}{cc}
c_{\beta} & -s_{\beta} \\
s_{\beta} & c_{\beta}
\end{array}\right) \cdot\left(\begin{array}{c}
G^{ \pm} \\
H^{ \pm}
\end{array}\right)
$$

\footnotetext{
${ }^{5}$ See early refs. [74-76] for the Rubakov-Callan effects.
} 
Similarly, two pseudoscalars of $\left(A_{1}, A_{2}\right)$ are transformed into neutral Nambu-Goldstone bosons $G^{0}$ and a pseudoscalar $A^{0}$ by

$$
\left(\begin{array}{l}
A_{1} \\
A_{2}
\end{array}\right)=\left(\begin{array}{cc}
c_{\beta} & -s_{\beta} \\
s_{\beta} & c_{\beta}
\end{array}\right) \cdot\left(\begin{array}{c}
G^{0} \\
A^{0}
\end{array}\right) .
$$

In the basis of $\left(H_{1}, H_{2}, A^{0}\right)$, the $3 \times 3$ mass squared matrix for the neutral states are diagonalized by $[38,71,80]$

$$
\begin{aligned}
& \left(\begin{array}{l}
h_{1} \\
h_{2} \\
h_{3}
\end{array}\right)=\mathcal{R} \cdot\left(\begin{array}{l}
H_{1} \\
H_{2} \\
A^{0}
\end{array}\right), \\
& \mathcal{M}_{0}^{2}=\mathcal{R}^{T} \cdot\left(\begin{array}{ccc}
m_{1}^{2} & 0 & 0 \\
0 & m_{2}^{2} & 0 \\
0 & 0 & m_{3}^{2}
\end{array}\right) \cdot \mathcal{R} \\
& \mathcal{R}=\mathcal{R}_{23}\left(\alpha_{c}\right) \cdot \mathcal{R}_{13}\left(\alpha_{b}\right) \cdot \mathcal{R}_{12}\left(\alpha+\frac{\pi}{2}\right) \\
& =\left(\begin{array}{ccc}
1 & 0 & 0 \\
0 & c_{\alpha_{c}} & s_{\alpha_{c}} \\
0 & -s_{\alpha_{c}} & c_{\alpha_{c}}
\end{array}\right) \cdot\left(\begin{array}{ccc}
c_{\alpha_{b}} & 0 & s_{\alpha_{b}} \\
0 & 1 & 0 \\
-s_{\alpha_{b}} & 0 & c_{\alpha_{b}}
\end{array}\right) \cdot\left(\begin{array}{ccc}
-s_{\alpha} & c_{\alpha} & 0 \\
-c_{\alpha} & -s_{\alpha} & 0 \\
0 & 0 & 1
\end{array}\right) \\
& =\left(\begin{array}{ccc}
-s_{\alpha} c_{\alpha_{b}} & c_{\alpha} c_{\alpha_{b}} & s_{\alpha_{b}} \\
s_{\alpha} s_{\alpha_{b}} s_{\alpha_{c}}-c_{\alpha} c_{\alpha_{c}} & -s_{\alpha} c_{\alpha_{c}}-c_{\alpha} s_{\alpha_{b}} s_{\alpha_{c}} & c_{\alpha_{b}} s_{\alpha_{c}} \\
s_{\alpha} s_{\alpha_{b}} c_{\alpha_{c}}+c_{\alpha} s_{\alpha_{c}} & s_{\alpha} s_{\alpha_{c}}-c_{\alpha} s_{\alpha_{b}} c_{\alpha_{c}} & c_{\alpha_{b}} c_{\alpha_{c}}
\end{array}\right) .
\end{aligned}
$$

Each term in the mass squared matrix of $\mathcal{M}_{0}^{2}$ is listed below

$$
\begin{aligned}
\mathcal{M}_{0}^{2} / v^{2} & =\left(\begin{array}{lll}
\hat{\mu}_{11}^{2} & \hat{\mu}_{12}^{2} & \hat{\mu}_{1 A}^{2} \\
\hat{\mu}_{12}^{2} & \hat{\mu}_{22}^{2} & \hat{\mu}_{2 A}^{2} \\
\hat{\mu}_{1 A}^{2} & \hat{\mu}_{2 A}^{2} & \hat{\mu}_{A A}^{2}
\end{array}\right), \\
\hat{\mu}_{11}^{2} & =\lambda_{1} c_{\beta}^{2}+\operatorname{Re} \lambda_{5} s_{\beta}^{2} c_{\theta}^{2}, \\
\hat{\mu}_{22}^{2} & =\lambda_{2} s_{\beta}^{2}+\operatorname{Re} \lambda_{5} c_{\beta}^{2} c_{\theta}^{2}, \\
\hat{\mu}_{12}^{2} & =\left(\lambda_{3}+\lambda_{4}-\operatorname{Re} \lambda_{5} s_{\theta}^{2}\right) s_{\beta} c_{\beta}, \\
\hat{\mu}_{1 A}^{2} & =-\frac{1}{2} \operatorname{Re} \lambda_{5} s_{2 \theta} s_{\beta}, \\
\hat{\mu}_{2 A}^{2} & =-\frac{1}{2} \operatorname{Re} \lambda_{5} s_{2 \theta} c_{\beta}, \\
\hat{\mu}_{A A}^{2} & =\operatorname{Re} \lambda_{5} s_{\theta}^{2} .
\end{aligned}
$$

In turn, the quartic scalar self couplings are expressed in terms of the Higgs boson masses and mixings as

$$
\lambda_{1}=\frac{1}{c_{\beta}^{2}} \sum_{i=1}^{3} \frac{m_{i}^{2}}{v^{2}}\left(\mathcal{R}_{i 1}^{2}-\frac{s_{\beta}^{2}}{t_{\theta}^{2}} \mathcal{R}_{i 3}^{2}\right),
$$




$$
\begin{aligned}
\lambda_{2} & =\frac{1}{s_{\beta}^{2}} \sum_{i=1}^{3} \frac{m_{i}^{2}}{v^{2}}\left(\mathcal{R}_{i 2}^{2}-\frac{c_{\beta}^{2}}{t_{\theta}^{2}} \mathcal{R}_{i 3}^{2}\right), \\
\lambda_{3} & =\sum_{i=1}^{3} \frac{m_{i}^{2}}{v^{2}}\left(\frac{1}{s_{\beta} c_{\beta}} \mathcal{R}_{i 1} \mathcal{R}_{i 2}-\frac{1}{t_{\theta}^{2}} \mathcal{R}_{i 3}^{2}\right)+\frac{2 m_{ \pm}^{2}}{v^{2}} \\
\lambda_{4} & =\frac{1}{s_{\theta}^{2}} \sum_{i} \frac{m_{i}^{2}}{v^{2}} \mathcal{R}_{i 3}^{2}-\frac{2 m_{ \pm}^{2}}{v^{2}} \\
\operatorname{Re} \lambda_{5} & =\frac{1}{s_{\theta}^{2}} \sum_{i} \frac{m_{i}^{2}}{v^{2}} \mathcal{R}_{i 3}^{2},
\end{aligned}
$$

in the SCPV scenario.

There is a well-known constraint from the $\left(\mathcal{M}_{0}^{2}\right)_{13}$ and $\left(\mathcal{M}_{0}^{2}\right)_{13}$ terms in eq. (A.6):

$$
\left(\mathcal{M}_{0}^{2}\right)_{13}=t_{\beta}\left(\mathcal{M}_{0}^{2}\right)_{23} \text {. }
$$

This leads to one additional constraint between mixing angles and mass eigenvalues as follows [81]

$$
\begin{aligned}
& \left(m_{1}^{2}-m_{2}^{2} s_{\alpha_{c}}^{2}-m_{3}^{2} c_{\alpha_{c}}^{2}\right) s_{\alpha_{b}}\left(t_{\alpha}+t_{\beta}\right)=\left(m_{2}^{2}-m_{3}^{2}\right)\left(t_{\alpha} t_{\beta}-1\right) s_{\alpha_{c}} c_{\alpha_{c}} \\
\Rightarrow & s_{\alpha_{b}}=\frac{\left(m_{2}^{2}-m_{3}^{2}\right)\left(t_{\alpha} t_{\beta}-1\right) s_{\alpha_{c}} c_{\alpha_{c}}}{\left(m_{1}^{2}-m_{2}^{2} s_{\alpha_{c}}^{2}-m_{3}^{2} c_{\alpha_{c}}^{2}\right)\left(t_{\alpha}+t_{\beta}\right)} .
\end{aligned}
$$

In practice, we use three mixing angles of $\left(\alpha, \alpha_{c}, \beta\right)$ as inputs. With the special limit of $\beta-\alpha=\pi / 2$, we have

$$
\begin{aligned}
& \left(m_{1}^{2}-m_{2}^{2} s_{\alpha_{c}}^{2}-m_{3}^{2} c_{\alpha_{c}}^{2}\right) s_{\alpha_{b}}=\left(m_{2}^{2}-m_{3}^{2}\right) s_{\alpha_{c}} c_{\alpha_{c}} t_{2 \beta} \\
\Rightarrow & s_{\alpha_{b}}=\frac{\left(m_{2}^{2}-m_{3}^{2}\right) s_{\alpha_{c}} c_{\alpha_{c}} t_{2 \beta}}{m_{1}^{2}-m_{2}^{2} s_{\alpha_{c}}^{2}-m_{3}^{2} c_{\alpha_{c}}^{2}},
\end{aligned}
$$

and there is a singularity at $t_{\beta}=1$ under this limit. In the small $\alpha_{c}$ limit, we can further approximate this relation as

$$
\alpha_{b} \approx \frac{m_{3}^{2}-m_{2}^{2}}{m_{3}^{2}-m_{1}^{2}} t_{2 \beta} \alpha_{c} .
$$

In the physical basis, the SCPV phase of $\theta$ is obtained from the relation of

$$
\frac{\hat{\mu}_{A A}^{2}}{\hat{\mu}_{1 A}^{2}}=-\frac{s_{\beta}}{t_{\theta}} \Rightarrow t_{\theta}=-s_{\beta} \frac{\sum_{i} m_{i}^{2} \mathcal{R}_{i 3}^{2}}{\sum_{i} m_{i}^{2} \mathcal{R}_{i 1} \mathcal{R}_{i 3}} .
$$

One may express $\hat{\mu}_{1 A}^{2}$ and $\hat{\mu}_{A A}^{2}$ in terms of masses and mixing angles explicitly as follows

$$
\begin{aligned}
& \hat{\mu}_{1 A}^{2} v^{2}=\left(m_{2}^{2} s_{\alpha_{c}}^{2}+m_{3}^{2} c_{\alpha_{c}}^{2}-m_{1}^{2}\right) s_{\alpha} s_{\alpha_{b}} c_{\alpha_{b}}+\left(m_{3}^{2}-m_{2}^{2}\right) c_{\alpha} c_{\alpha_{b}} s_{\alpha_{c}} c_{\alpha_{c}}, \\
& \hat{\mu}_{A A}^{2} v^{2}=m_{1}^{2} s_{\alpha_{b}}^{2}+\left(m_{2}^{2} s_{\alpha_{c}}^{2}+m_{3}^{2} c_{\alpha_{c}}^{2}\right) c_{\alpha_{b}}^{2} .
\end{aligned}
$$

By combining with the dependences of $\left|\alpha_{b}\right|$ on $\alpha_{c}$ as depicted in figure 1 , the $\hat{\mu}_{1 A}^{2}$ and $\hat{\mu}_{A A}^{2}$ become

$$
\hat{\mu}_{1 A}^{2} \sim-\frac{m_{3}^{2}-m_{2}^{2}}{v^{2}} \frac{s_{\beta} \alpha_{c}}{c_{2 \beta}}, \quad \hat{\mu}_{A A}^{2} \sim \frac{m_{3}^{2}}{v^{2}}
$$




\begin{tabular}{|c|c|}
\hline Generical basis & Physical basis \\
\hline$\lambda_{1,2,3,4}, \operatorname{Re} \lambda_{5}$ & $m_{1,2,3}, m_{ \pm}, v$ \\
$m_{11}^{2}, m_{22}^{2}, \operatorname{Re} m_{12}^{2}$ & $\alpha, \alpha_{b}, \alpha_{c}, \beta, \theta$ \\
\hline
\end{tabular}

Table 1. The parameter inputs in both the generical basis and the physical basis for the SCPV scenario.

under the $\beta-\alpha=\pi / 2$ and small $\alpha_{c}$ limit. Here, we have also made used of eq. (A.11). Clearly, one can envision that $\hat{\mu}_{1 A}^{2} \ll \hat{\mu}_{A A}^{2}$. Accordingly, the SCPV phase becomes

$$
t_{\theta} \approx \frac{m_{3}^{2} c_{2 \beta}}{\left(m_{3}^{2}-m_{2}^{2}\right) \alpha_{c}} .
$$

Obviously, the solutions of the SCPV are approaching to $\pm \frac{\pi}{2}$ when the CPV mixing angle $\alpha_{c}$ is suppressed and/or two heavy neutral Higgs bosons are very mass degenerate.

We summarize the parameter inputs for the SCPV scenario in both the physical basis and the generical basis in table. 1 . The CPV mixing angle $\alpha_{b}$ and the SCPV phase $\theta$ can be obtained by eq. (A.9) and eq. (A.12), respectively. Therefore, one has eight independent parameters in both basis.

\section{A.2 The mass spectrum and self couplings with the ECPV}

The most general $2 \mathrm{HDM}$ potential with complex parameters of $m_{12}^{2}$ and $\lambda_{5}$ violates the CP symmetry explicitly. The corresponding minimization conditions were previously given in eqs. (2.3).

For the mass spectrum, the same conventions of the mass squared matrix and mixing angles are adopted as in the SCPV scenario. The charged Higgs boson masses are expressed as

$$
m_{ \pm}^{2}=-\frac{1}{2}\left(\lambda_{4}-\operatorname{Re} \lambda_{5}-\operatorname{Im} \lambda_{5} / t_{\theta}\right) v^{2}-\frac{\operatorname{Im} m_{12}^{2}}{s_{\beta} c_{\beta} s_{\theta}}
$$

Schematically, the neutral mass squared matrix is the same as in eq. (A.6a), with each element listed as below

$$
\begin{aligned}
\hat{\mu}_{11}^{2} & =\lambda_{1} c_{\beta}^{2}+\operatorname{Re} \lambda_{5} c_{\theta}^{2} s_{\beta}^{2}+\operatorname{Im} \lambda_{5} \frac{c_{2 \theta} s_{\beta}^{2}}{2 t_{\theta}}-\frac{\operatorname{Im} m_{12}^{2} t_{\beta}}{v^{2} s_{\theta}} \\
\hat{\mu}_{22}^{2} & =\lambda_{2} s_{\beta}^{2}+\operatorname{Re} \lambda_{5} c_{\theta}^{2} c_{\beta}^{2}+\operatorname{Im} \lambda_{5} \frac{c_{2 \theta} c_{\beta}^{2}}{2 t_{\theta}}-\frac{\operatorname{Im} m_{12}^{2}}{v^{2} t_{\beta} s_{\theta}} \\
\hat{\mu}_{12}^{2} & =\left(\lambda_{3}+\lambda_{4}-\operatorname{Re} \lambda_{5} s_{\theta}^{2}-\operatorname{Im} \lambda_{5} \frac{1+2 s_{\theta}^{2}}{2 t_{\theta}}\right) s_{\beta} c_{\beta}+\frac{\operatorname{Im} m_{12}^{2}}{v^{2} s_{\theta}} \\
\hat{\mu}_{1 A}^{2} & =-\frac{1}{2}\left(\operatorname{Re} \lambda_{5} s_{2 \theta}+\operatorname{Im} \lambda_{5} c_{2 \theta}\right) s_{\beta} \\
\hat{\mu}_{2 A}^{2} & =-\frac{1}{2}\left(\operatorname{Re} \lambda_{5} s_{2 \theta}+\operatorname{Im} \lambda_{5} c_{2 \theta}\right) c_{\beta} \\
\hat{\mu}_{A A}^{2} & =\operatorname{Re} \lambda_{5} s_{\theta}^{2}+\operatorname{Im} \lambda_{5} \frac{1+2 s_{\theta}^{2}}{2 t_{\theta}}-\frac{\operatorname{Im} m_{12}^{2}}{v^{2} s_{\beta} c_{\beta} s_{\theta}}
\end{aligned}
$$


From the expressions of $\hat{\mu}_{1 A}^{2}$ and $\hat{\mu}_{A A}^{2}$, one finds the relation between the imaginary components of $\operatorname{Im} m_{12}^{2}$ and $\operatorname{Im} \lambda_{5}$ as

$$
\hat{\mu}_{A A}^{2} s_{\theta} c_{\theta}+\frac{\hat{\mu}_{1 A}^{2}}{s_{\beta}} s_{\theta}^{2}=\frac{1}{2} \operatorname{Im} \lambda_{5}-\frac{\operatorname{Im} m_{12}^{2}}{v^{2} s_{\beta} c_{\beta}} c_{\theta},
$$

which will be used for solving the relative CPV phase of $\theta$ in general when replacing $\hat{\mu}_{A A}^{2}$ and $\hat{\mu}_{1 A}^{2}$ by physical inputs according to eq. (A.5).

In the ECPV scenario, the phase transformation of $\Phi_{2}$ can remove one of the three phases. The third minimization condition in eq. (2.3c) will help to relate the two remaining phases. Thus, we have only one free CPV phase again. There are several situations in solving the CPV phase of $\theta$ from eq. (A.18) for the ECPV scenario:

1. $\operatorname{Im} m_{12}^{2}=0$, the above equation is a quadratic equation of $t_{\theta}$. This is equivalent to rephase $\Phi_{2}$ as $\Phi_{2} \rightarrow e^{-i \delta_{1}} \Phi_{2}$.

2. $\operatorname{Im} \lambda_{5}=0$, the above equation is a quartic equation of $s_{\theta}$. This is equivalent to rephase $\Phi_{2}$ as $\Phi_{2} \rightarrow e^{-i \delta_{2} / 2} \Phi_{2}$.

3. If one keeps both $\operatorname{Im} m_{12}^{2}$ and $\operatorname{Im} \lambda_{5}$ non-zero, the above equation is a quartic equation of $t_{\theta / 2}$.

To simplify our discussions, we take the special case of $\operatorname{Im} m_{12}^{2}=0$, where $t_{\theta}$ satisfies

$$
\left(\frac{2 \hat{\mu}_{1 A}^{2}}{s_{\beta}}-\operatorname{Im} \lambda_{5}\right) t_{\theta}^{2}+2 \hat{\mu}_{A A}^{2} t_{\theta}-\operatorname{Im} \lambda_{5}=0 .
$$

The exact solution for $t_{\theta}$ is expressed as

$$
t_{\theta}=\frac{1}{2 \hat{\mu}_{1 A}^{2} / s_{\beta}-\operatorname{Im} \lambda_{5}}\left[\mp \sqrt{\left(\hat{\mu}_{A A}^{2}\right)^{2}+\operatorname{Im} \lambda_{5}\left(2 \hat{\mu}_{1 A}^{2} / s_{\beta}-\operatorname{Im} \lambda_{5}\right)}-\hat{\mu}_{A A}^{2}\right] .
$$

Given that we have $\hat{\mu}_{1 A}^{2} \ll \hat{\mu}_{A A}^{2}$ in the small $\alpha_{c}$ limit, the $\mp$ signs in the exact solutions in eq. (A.20) correspond to the limits of $\theta \rightarrow \pm \pi / 2$ and $\theta \rightarrow 0$, respectively. To restore the exact solution of $t_{\theta}$ in the SCPV scenario, one should take the - sign in eq. (A.20). It turns out that the relative CPV phase $\theta$ will enter into the biased term to make the domain wall collapse in the form of $s_{2 \theta}$. In both limit of $\theta \rightarrow \pm \pi / 2$, we have $s_{2 \theta} \rightarrow 0$.

Besides of the CPV mixing angle of $\alpha_{c}$, the ECPV parameter of $\operatorname{Im} \lambda_{5}$ also plays a role. In the limit of $\left|\operatorname{Im} \lambda_{5}\right| \ll \hat{\mu}_{1 A}^{2} / s_{\beta}$, we have

$$
t_{\theta} \approx-s_{\beta} \frac{\hat{\mu}_{A A}^{2}}{\hat{\mu}_{1 A}^{2}}-\frac{\operatorname{Im} \lambda_{5} s_{\beta}}{2 \hat{\mu}_{1 A}^{2}}\left(\frac{s_{\beta} \hat{\mu}_{A A}^{2}}{\hat{\mu}_{1 A}^{2}}+\frac{\hat{\mu}_{1 A}^{2}}{s_{\beta} \hat{\mu}_{A A}^{2}}\right)+\mathcal{O}\left(\left(\operatorname{Im} \lambda_{5}\right)^{2}\right),
$$

with the leading contributions the same as the expression in the SCPV scenario eq. (A.12). Correspondingly, the relative CPV phase of $\theta$ is expected to depend on CPV mixing angle $\alpha_{c}$ significantly. On the other hand, in the limit of $\hat{\mu}_{A A}^{2} \gg\left|\operatorname{Im} \lambda_{5}\right| \gg \hat{\mu}_{1 A}^{2} / s_{\beta}$, we have the approximation to $t_{\theta}$ as

$$
t_{\theta} \approx \frac{2 \hat{\mu}_{A A}^{2}}{\operatorname{Im} \lambda_{5}}-\frac{\operatorname{Im} \lambda_{5}}{2 \hat{\mu}_{A A}^{2}}+\mathcal{O}\left(\left(\operatorname{Im} \lambda_{5}\right)^{2}\right)
$$




\begin{tabular}{|c|c|}
\hline Generical basis & Physical basis \\
\hline$\lambda_{1,2,3,4}, \operatorname{Re} \lambda_{5}, \operatorname{Im} \lambda_{5}$ & $m_{1,2,3}, m_{ \pm}, v$ \\
$m_{11}^{2}, m_{22}^{2}, \operatorname{Re} m_{12}^{2}, \operatorname{Im} m_{12}^{2}$ & $\alpha, \alpha_{b}, \alpha_{c}, \beta, \theta, \operatorname{Im} \lambda_{5}, \operatorname{Im} m_{12}^{2}$ \\
\hline
\end{tabular}

Table 2. The parameter inputs in both the generical basis and the physical basis for the ECPV scenario.

As we have shown above that $\hat{\mu}_{A A}^{2} \rightarrow m_{3}^{2} / v^{2}$ in the small $\alpha_{c}$ limit, the value of $\theta$ will be independent of the CPV mixing angle $\alpha_{c}$ in such a limit.

With the solution of $\theta$ obtained in eq. (A.20), we can solve for the quartic scalar self couplings in terms of the Higgs boson masses and mixings as below

$$
\begin{aligned}
\lambda_{1} & =\sum_{i=1}^{3} \frac{m_{i}^{2}}{v^{2} c_{\beta}^{2}}\left(\mathcal{R}_{i 1}^{2}-\frac{s_{\beta}^{2}}{t_{\theta}^{2}} \mathcal{R}_{i 3}^{2}\right)+\frac{\operatorname{Im} \lambda_{5} t_{\beta}^{2}}{2 t_{\theta} s_{\theta}^{2}} \\
\lambda_{2} & =\sum_{i=1}^{3} \frac{m_{i}^{2}}{v^{2} s_{\beta}^{2}}\left(\mathcal{R}_{i 2}^{2}-\frac{c_{\beta}^{2}}{t_{\theta}^{2}} \mathcal{R}_{i 3}^{2}\right)+\frac{\operatorname{Im} \lambda_{5}}{2 t_{\beta}^{2} t_{\theta} s_{\theta}^{2}} \\
\lambda_{3} & =\sum_{i=1}^{3} \frac{m_{i}^{2}}{v^{2} s_{\beta} c_{\beta}}\left(\mathcal{R}_{i 1} \mathcal{R}_{i 2}-\frac{s_{\beta} c_{\beta}}{t_{\theta}^{2}} \mathcal{R}_{i 3}^{2}\right)+\frac{2 m_{ \pm}^{2}}{v^{2}}+\frac{\operatorname{Im} \lambda_{5}}{2 t_{\theta} s_{\theta}^{2}} \\
\lambda_{4} & =\sum_{i=1}^{3} \frac{m_{i}^{2} \mathcal{R}_{i 3}^{2}}{v^{2} s_{\theta}^{2}}-\frac{2 m_{ \pm}^{2}}{v^{2}}-\frac{\operatorname{Im} \lambda_{5}}{2 t_{\theta} s_{\theta}^{2}} \\
\operatorname{Re} \lambda_{5} & =\sum_{i=1}^{3} \frac{m_{i}^{2} \mathcal{R}_{i 3}^{2}}{v^{2} s_{\theta}^{2}}-\operatorname{Im} \lambda_{5} \frac{1+2 s_{\theta}^{2}}{2 t_{\theta} s_{\theta}^{2}}
\end{aligned}
$$

with the choice of $\operatorname{Im} m_{12}^{2}=0$. All quartic scalar self couplings can easily return to those in the SCPV scenario as listed in eqs. (A.7), by taking $\operatorname{Im} \lambda_{5}=0$. The Higgs potential with the ECPV scenario can be extended from eq. (2.9) as below

$$
\begin{aligned}
V & =V_{\mathrm{CPC}}+V_{\mathrm{CPV}}, \\
V_{\mathrm{CPV}} & =V_{\mathrm{SCPV}}+V_{\mathrm{ECPV}} \\
V_{\mathrm{SCPV}} & =\left(-\frac{\operatorname{Re} m_{12}^{2}}{v^{2} s_{\beta} c_{\beta}} c_{\theta}+\frac{1}{4} \operatorname{Re} \lambda_{5} c_{2 \theta}\right) v^{4} s_{\beta}^{2} c_{\beta}^{2}, \\
V_{\mathrm{ECPV}} & =-\frac{1}{4} \operatorname{Im} \lambda_{5} s_{2 \theta} v^{4} s_{\beta}^{2} c_{\beta}^{2} .
\end{aligned}
$$

We summarize the parameter inputs for the ECPV scenario in both the physical basis and the generical basis in table. 2. The CPV mixing angle $\alpha_{b}$ is obtained from eq. (A.9) from other masses and mixing angles. The relative CP phase can be obtained from eq. (A.20). As we have argued previously, one can always remove one of two ECPV parameters by rephasing the second doublet $\Phi_{2}$. Without loss of generality, we choose to take $\operatorname{Im} m_{12}^{2}=0$. Hence, one has nine independent input parameters in both basis. We shall describe the procedures of converting the input parameters in the physical basis to the generic basis. 


\begin{tabular}{|c|c|c|}
\hline & Type-I & Type-II \\
\hline $\begin{array}{c}c_{u, 1} \\
c_{d, 1}=c_{\ell, 1} \\
\tilde{c}_{u, 1} \\
\tilde{c}_{d, 1}=\tilde{c}_{\ell, 1} \\
a_{1}\end{array}$ & $\begin{array}{c}\mathcal{R}_{12} / s_{\beta} \rightarrow c_{\alpha_{b}} \\
\mathcal{R}_{12} / s_{\beta} \rightarrow c_{\alpha_{b}} \\
-\mathcal{R}_{13} / t_{\beta} \rightarrow-s_{\alpha_{b}} / t_{\beta} \\
\mathcal{R}_{13} / t_{\beta} \rightarrow s_{\alpha_{b}} / t_{\beta} \\
\mathcal{R}_{12} s_{\beta}+\mathcal{R}_{11} c_{\beta} \rightarrow c_{\alpha_{b}}\end{array}$ & $\begin{array}{c}\mathcal{R}_{12} / s_{\beta} \rightarrow c_{\alpha_{b}} \\
\mathcal{R}_{12} / s_{\beta} \rightarrow c_{\alpha_{b}} \\
-\mathcal{R}_{13} / t_{\beta} \rightarrow-s_{\alpha_{b}} / t_{\beta} \\
-\mathcal{R}_{13} t_{\beta} \rightarrow-s_{\alpha_{b}} t_{\beta} \\
\mathcal{R}_{12} s_{\beta}+\mathcal{R}_{11} c_{\beta} \rightarrow c_{\alpha_{b}}\end{array}$ \\
\hline $\begin{array}{c}c_{u, 2} \\
c_{d, 2}=c_{\ell, 2} \\
\tilde{c}_{u, 2} \\
\tilde{c}_{d, 2}=\tilde{c}_{\ell, 2} \\
a_{2}\end{array}$ & $\begin{array}{c}\mathcal{R}_{22} / s_{\beta} \rightarrow c_{\alpha_{c}} / t_{\beta}-s_{\alpha_{b}} s_{\alpha_{c}} \\
\mathcal{R}_{22} / s_{\beta} \rightarrow c_{\alpha_{c}} / t_{\beta}-s_{\alpha_{b}} s_{\alpha_{c}} \\
-\mathcal{R}_{23} / t_{\beta} \rightarrow-c_{\alpha_{b}} s_{\alpha_{c}} / t_{\beta} \\
\mathcal{R}_{23} / t_{\beta} \rightarrow c_{\alpha_{b}} s_{\alpha_{c}} / t_{\beta} \\
\mathcal{R}_{22} s_{\beta}+\mathcal{R}_{21} c_{\beta} \rightarrow-s_{\alpha_{b}} s_{\alpha_{c}}\end{array}$ & $\begin{array}{c}\mathcal{R}_{22} / s_{\beta} \rightarrow c_{\alpha_{c}} / t_{\beta}-s_{\alpha_{b}} s_{\alpha_{c}} \\
\mathcal{R}_{21} / c_{\beta} \rightarrow-s_{\alpha_{b}} s_{\alpha_{c}}-c_{\alpha_{c}} t_{\beta} \\
-\mathcal{R}_{23} / t_{\beta} \rightarrow-c_{\alpha_{b}} s_{\alpha_{c}} / t_{\beta} \\
-\mathcal{R}_{23} t_{\beta} \rightarrow-c_{\alpha_{b}} s_{\alpha_{c}} t_{\beta} \\
\mathcal{R}_{22} s_{\beta}+\mathcal{R}_{21} c_{\beta} \rightarrow-s_{\alpha_{b}} s_{\alpha_{c}}\end{array}$ \\
\hline $\begin{array}{c}c_{u, 3} \\
c_{d, 3}=c_{\ell, 3} \\
\tilde{c}_{u, 3} \\
\tilde{c}_{d, 3}=\tilde{c}_{\ell, 3} \\
a_{3}\end{array}$ & $\begin{array}{c}\mathcal{R}_{32} / s_{\beta} \rightarrow-s_{\alpha_{c}} / t_{\beta}-s_{\alpha_{b}} c_{\alpha_{c}} \\
\mathcal{R}_{31} / s_{\beta} \rightarrow-s_{\alpha_{c}} / t_{\beta}-s_{\alpha_{b}} c_{\alpha_{c}} \\
-\mathcal{R}_{33} / t_{\beta} \rightarrow-c_{\alpha_{b}} c_{\alpha_{c}} / t_{\beta} \\
\mathcal{R}_{33} / t_{\beta} \rightarrow c_{\alpha_{b}} c_{\alpha_{c}} / t_{\beta} \\
\mathcal{R}_{32} s_{\beta}+\mathcal{R}_{31} c_{\beta} \rightarrow-s_{\alpha_{b}} c_{\alpha_{c}}\end{array}$ & $\begin{array}{c}\mathcal{R}_{32} / s_{\beta} \rightarrow-s_{\alpha_{c}} / t_{\beta}-s_{\alpha_{b}} c_{\alpha_{c}} \\
\mathcal{R}_{31} / c_{\beta} \rightarrow-s_{\alpha_{b}} c_{\alpha_{c}}+s_{\alpha_{c}} t_{\beta} \\
-\mathcal{R}_{33} / t_{\beta} \rightarrow-c_{\alpha_{b}} c_{\alpha_{c}} / t_{\beta} \\
-\mathcal{R}_{33} t_{\beta} \rightarrow-c_{\alpha_{b}} c_{\alpha_{c}} t_{\beta} \\
\mathcal{R}_{32} s_{\beta}+\mathcal{R}_{31} c_{\beta} \rightarrow-s_{\alpha_{b}} c_{\alpha_{c}}\end{array}$ \\
\hline
\end{tabular}

Table 3. The SM fermion and gauge boson couplings to Higgs boson mass eigenstates of $h_{1,2,3}$, and their expressions in the limit of $\beta-\alpha=\pi / 2$.

1. In the physical basis, all necessary input parameters are masses of three neutral Higgs bosons $m_{1,2,3}$, the mass of charged Higgs bosons $m_{ \pm}$, the Higgs VEV of $v=$ $\left(\sqrt{2} G_{F}\right)^{-1 / 2} \simeq 246 \mathrm{GeV}$, and three mixing angles of $\left(\alpha, \alpha_{c}, \beta\right)$, as well as the ECPV parameter $\operatorname{Im} \lambda_{5}$. The state of $h_{1}$ will be regarded as the SM-like Higgs boson, with $m_{1}=125 \mathrm{GeV}$. To simplify the discussion, we shall take the special limit of $\alpha=\beta-\frac{\pi}{2}$.

2. The other CPV mixing angle $\alpha_{b}$ is related to three input mixing angles of $\left(\alpha, \alpha_{c}, \beta\right)$ from eq. (A.9), which is reduced to eq. (A.10) in the special limit of $\alpha=\beta-\frac{\pi}{2}$.

3. The relative CPV phase of $\theta$ is derived from the exact solution in eq. (A.20) with all above parameter plus the ECPV parameter of $\operatorname{Im} \lambda_{5}$.

4. With all Higgs masses of $m_{1,2,3, \pm}$, mixing angles of $\left(\alpha, \alpha_{b}, \alpha_{c}, \beta\right)$, the relative CPV phase of $\theta$, and the ECPV parameter of $\operatorname{Im} \lambda_{5}$, we obtain the quartic scalar self couplings from eqs. (A.23), and mass squared parameters of $\left(m_{11}^{2}, m_{22}^{2}, \operatorname{Re} m_{12}^{2}\right)$ from the minimization conditions in eqs. (2.3). These parameters in the generical basis will be used for solving the domain wall profiles and determine the sizes of the biased terms for the domain wall collapse.

\section{A.3 The Yukawa couplings in the CPV 2HDM}

We focus on the CPV $2 \mathrm{HDM}$ where the Yukawa sector has a $\mathbb{Z}_{2}$ symmetry and $\Phi_{1}$ and $\Phi_{2}$ each only gives mass to up-type quarks or down-type quarks and charged leptons. This is sufficient to suppress tree-level flavor changing processes mediated by the neutral Higgs 
bosons. The Yukawa couplings for the Type-I and Type-II 2HDM read (and suppressing the CKM mixing),

$$
\mathcal{L}= \begin{cases}-\left(\frac{c_{\alpha}}{s_{\beta}} \frac{m_{u}}{v}\right) \bar{Q}_{L} \tilde{\Phi}_{2} u_{R}-\left(\frac{c_{\alpha}}{s_{\beta}} \frac{m_{d}}{v}\right) \bar{Q}_{L} \Phi_{2} d_{R}+\text { h.c. } & \text { Type }-\mathrm{I} \\ -\left(\frac{c_{\alpha}}{s_{\beta}} \frac{m_{u}}{v}\right) \bar{Q}_{L} \tilde{\Phi}_{2} u_{R}+\left(\frac{s_{\alpha}}{c_{\beta}} \frac{m_{d}}{v}\right) \bar{Q}_{L} \Phi_{1} d_{R}+\text { h.c. } & \text { Type }-\mathrm{II},\end{cases}
$$

where $Q_{L}^{T}=\left(u_{L}, d_{L}\right)$ and $\tilde{\Phi}_{2} \equiv i \sigma_{2} \Phi_{2}^{*}$. For both cases, the charged lepton Yukawa coupling has the same form as that of the down-type quarks. Therefore, we can express the couplings between neutral Higgs bosons and the fermions and gauge bosons in the mass eigenbasis

$$
\mathcal{L}=\sum_{i=1}^{3}\left[-m_{f}\left(c_{f, i} \bar{f} f+\tilde{c}_{f, i} \bar{f} i \gamma_{5} f\right)+a_{i}\left(2 m_{W}^{2} W_{\mu} W^{\mu}+m_{Z}^{2} Z_{\mu} Z^{\mu}\right)\right] \frac{h_{i}}{v} .
$$

When $c_{f, i} \tilde{c}_{f, i} \neq 0$ or $a_{i} \tilde{c}_{f, i} \neq 0$, the mass eigenstate $h_{i}$ couples to both CP-even and CPodd operators, so the CP symmetry is violated. The coefficients of $c_{f, i}, \tilde{c}_{f, i}$ and $a_{i}$ can be derived from the elements of the rotation matrix $\mathcal{R}$ defined in eq. (A.5), which were also previously obtained in refs. [37, 38, 40]. Here, we summarize their explicit expressions in table. 3. In the special limit of $\beta-\alpha=\pi / 2$, the Yukawa couplings and Higgs gauge couplings are determined by the CPV mixing angles of $\left(\alpha_{b}, \alpha_{c}\right)$ and $t_{\beta}$. By taking the CPC limit of $\alpha_{b}=\alpha_{c}=0$, it is evident that $\left(h_{1}, h_{2}\right)$ have the purely CP-even Yukawa couplings of $c_{f, i}$, while $h_{3}$ has the purely CP-odd Yukawa couplings of $\tilde{c}_{f, i}$. The previous studies of the collider measurements of the CPV in the Higgs Yukawa couplings can be found in refs. [82-99].

Open Access. This article is distributed under the terms of the Creative Commons Attribution License (CC-BY 4.0), which permits any use, distribution and reproduction in any medium, provided the original author(s) and source are credited.

\section{References}

[1] ATLAS collaboration, Observation of a new particle in the search for the Standard Model Higgs boson with the ATLAS detector at the LHC, Phys. Lett. B 716 (2012) 1 [arXiv: 1207.7214] [INSPIRE].

[2] CMS collaboration, Observation of a new boson at a mass of $125 \mathrm{GeV}$ with the CMS experiment at the LHC, Phys. Lett. B 716 (2012) 30 [arXiv:1207.7235] [INSPIRE].

[3] A.D. Sakharov, Violation of CP Invariance, $C$ asymmetry, and baryon asymmetry of the universe, Sov. Phys. Usp. 34 (1991) 392 [Usp. Fiz. Nauk. 161 (1991) 61] [INSPIRE].

[4] T.W.B. Kibble, Topology of cosmic domains and strings, J. Phys. A 9 (1976) 1387 [INSPIRE].

[5] W.H. Zurek, Cosmological experiments in superfluid Helium?, Nature 317 (1985) 505 [INSPIRE].

[6] T. Vachaspati, Kinks and domain walls: an introduction to classical and quantum solitons, Cambridge University Press, Camrbidge U.K. (2010). 
[7] T.D. Lee, CP nonconservation and spontaneous symmetry breaking, Phys. Rept. 9 (1974) 143 [INSPIRE].

[8] S.-S. Bao and Y.-L. Wu, Neutral Higgs production on LHC in the two-Higgs-doublet model with spontaneous CP-violation, Phys. Rev. D 81 (2010) 075020 [arXiv:0907.3606] [INSPIRE].

[9] S. Ipek, Perturbative analysis of the electron electric dipole moment and CP-violation in two-Higgs-doublet models, Phys. Rev. D 89 (2014) 073012 [arXiv:1310.6790] [INSPIRE].

[10] B. Grzadkowski, O.M. Ogreid and P. Osland, Measuring CP-violation in Two-Higgs-Doublet models in light of the LHC Higgs data, JHEP 11 (2014) 084 [arXiv:1409.7265] [INSPIRE].

[11] M. Eto, M. Kurachi and M. Nitta, Constraints on two Higgs doublet models from domain walls, Phys. Lett. B 785 (2018) 447 [arXiv: 1803.04662] [InSPIRE].

[12] M. Eto, M. Kurachi and M. Nitta, Non-Abelian strings and domain walls in two Higgs doublet models, JHEP 08 (2018) 195 [arXiv: 1805.07015] [INSPIRE].

[13] Y. Zeldovich, I. Kobzarev and L.B. Okun, Cosmological Consequences of the Spontaneous Breakdown of Discrete Symmetry, Zh. Eksp. Teor. Fiz. 67 (1974) 3 [Sov. Phys. JETP 40 (1974) 1] [INSPIRE].

[14] A.H. Guth, The inflationary universe: a possible solution to the horizon and flatness problems, Adv. Ser. Astrophys. Cosmol. 3 (1987) 139.

[15] G. Lazarides and Q. Shafi, Axion models with no domain wall problem, Phys. Lett. B 115 (1982) 21 [INSPIRE].

[16] S.M. Barr, D.B. Reiss and A. Zee, Families, the invisible axion, and domain walls, Phys. Lett. B 116 (1982) 227 [INSPIRE].

[17] G. Lazarides, M. Reig, Q. Shafi, R. Srivastava and J.W.F. Valle, Spontaneous breaking of lepton number and the cosmological domain wall problem, Phys. Rev. Lett. 122 (2019) 151301 [arXiv:1806.11198] [INSPIRE].

[18] A. Vilenkin, Gravitational field of vacuum domain walls and strings, Phys. Rev. D 23 (1981) 852 [INSPIRE].

[19] G.B. Gelmini, M. Gleiser and E.W. Kolb, Cosmology of biased discrete symmetry breaking, Phys. Rev. D 39 (1989) 1558 [InSPIRE].

[20] S.E. Larsson, S. Sarkar and P.L. White, Evading the cosmological domain wall problem, Phys. Rev. D 55 (1997) 5129 [hep-ph/9608319] [INSPIRE].

[21] T. Hiramatsu, M. Kawasaki and K. Saikawa, Gravitational Waves from Collapsing Domain Walls, JCAP 05 (2010) 032 [arXiv: 1002.1555] [INSPIRE].

[22] M. Kawasaki and K. Saikawa, Study of gravitational radiation from cosmic domain walls, JCAP 09 (2011) 008 [arXiv:1102.5628] [INSPIRE].

[23] K. Saikawa, A review of gravitational waves from cosmic domain walls, Universe 3 (2017) 40 [arXiv: 1703.02576] [INSPIRE].

[24] R. Zhou, J. Yang and L. Bian, Gravitational waves from first-order phase transition and domain wall, JHEP 04 (2020) 071 [arXiv:2001.04741] [INSPIRE].

[25] N. Chen, T. Li and Y. Wu, The gravitational waves from the collapsing domain walls in the complex singlet model, JHEP 08 (2020) 117 [arXiv:2004.10148] [INSPIRE]. 
[26] J. Jaeckel, S. Schenk and M. Spannowsky, Probing dark matter clumps, strings and domain walls with gravitational wave detectors, arXiv:2004.13724 [INSPIRE].

[27] P. Amaro-Seoane et al., eLISA/NGO: astrophysics and cosmology in the gravitational-wave milli-Hertz regime, GW Notes 6 (2013) 4 [arXiv:1201.3621] [INSPIRE].

[28] P. Amaro-Seoane et al., Low-frequency gravitational-wave science with eLISA/NGO, Class. Quant. Grav. 29 (2012) 124016 [arXiv: 1202.0839] [INSPIRE].

[29] W.-H. Ruan, Z.-K. Guo, R.-G. Cai and Y.-Z. Zhang, Taiji program: gravitational-wave sources, Int. J. Mod. Phys. A 35 (2020) 2050075 [arXiv:1807.09495] [INSPIRE].

[30] TianQIN collaboration, TianQin: a space-borne gravitational wave detector, Class. Quant. Grav. 33 (2016) 035010 [arXiv: 1512.02076] [INSPIRE].

[31] G. Janssen et al., Gravitational wave astronomy with the SKA, PoS (AASKA14) 037 [arXiv: 1501.00127] [INSPIRE].

[32] S. Kawamura et al., The Japanese space gravitational wave antenna: DECIGO, Class. Quant. Grav. 28 (2011) 094011 [inSPIRE].

[33] ACME collaboration, Order of magnitude smaller limit on the electric dipole moment of the Electron, Science 343 (2014) 269 [arXiv:1310.7534] [INSPIRE].

[34] ACME collaboration, Improved limit on the electric dipole moment of the electron, Nature 562 (2018) 355 [INSPIRE].

[35] B. Graner, Y. Chen, E.G. Lindahl and B.R. Heckel, Reduced Limit on the Permanent Electric Dipole Moment of Hg199, Phys. Rev. Lett. 116 (2016) 161601 [Erratum ibid. 119 (2017) 119901] [arXiv: 1601.04339] [INSPIRE].

[36] M. Bishof et al., Improved limit on the ${ }^{225}$ Ra electric dipole moment, Phys. Rev. C 94 (2016) 025501 [arXiv: 1606.04931] [INSPIRE].

[37] J. Shu and Y. Zhang, Impact of a CP-violating Higgs sector: from LHC to baryogenesis, Phys. Rev. Lett. 111 (2013) 091801 [arXiv: 1304.0773] [INSPIRE].

[38] S. Inoue, M.J. Ramsey-Musolf and Y. Zhang, CP-violating phenomenology of flavor conserving two Higgs doublet models, Phys. Rev. D 89 (2014) 115023 [arXiv:1403.4257] [INSPIRE].

[39] L. Bian, T. Liu and J. Shu, Cancellations between two-loop contributions to the electron electric dipole moment with a CP-violating Higgs sector, Phys. Rev. Lett. 115 (2015) 021801 [arXiv:1411.6695] [INSPIRE].

[40] C.-Y. Chen, S. Dawson and Y. Zhang, Complementarity of LHC and EDMs for exploring Higgs CP-violation, JHEP 06 (2015) 056 [arXiv: 1503.01114] [INSPIRE].

[41] L. Bian and N. Chen, Cancellation mechanism in the predictions of electric dipole moments, Phys. Rev. D 95 (2017) 115029 [arXiv:1608.07975] [INSPIRE].

[42] D. Egana-Ugrinovic and S. Thomas, Higgs boson contributions to the electron electric dipole moment, arXiv:1810.08631 [INSPIRE].

[43] E.J. Chun, J. Kim and T. Mondal, Electron EDM and muon anomalous magnetic moment in two-Higgs-doublet models, JHEP 12 (2019) 068 [arXiv:1906.00612] [INSPIRE].

[44] K. Cheung, A. Jueid, Y.-N. Mao and S. Moretti, The 2-Higgs-doublet model with soft CP-violation confronting electric dipole moments and colliders, arXiv:2003.04178 [INSPIRE]. 
[45] S. Kanemura, M. Kubota and K. Yagyu, Aligned CP-violating Higgs sector canceling the electric dipole moment, JHEP 08 (2020) 026 [arXiv: 2004.03943] [INSPIRE].

[46] C. Cesarotti, Q. Lu, Y. Nakai, A. Parikh and M. Reece, Interpreting the electron EDM constraint, JHEP 05 (2019) 059 [arXiv: 1810.07736] [INSPIRE].

[47] S. Davidson and H.E. Haber, Basis-independent methods for the two-Higgs-doublet model, Phys. Rev. D 72 (2005) 035004 [Erratum ibid. 72 (2005) 099902] [hep-ph/0504050] [INSPIRE].

[48] J.F. Gunion and H.E. Haber, Conditions for CP-violation in the general two-Higgs-doublet model, Phys. Rev. D 72 (2005) 095002 [hep-ph/0506227] [INSPIRE].

[49] I.P. Ivanov, Minkowski space structure of the Higgs potential in 2HDM, Phys. Rev. D 75 (2007) 035001 [Erratum ibid. 76 (2007) 039902] [hep-ph/0609018] [INSPIRE].

[50] I.P. Ivanov, Minkowski space structure of the Higgs potential in 2HDM. II. Minima, symmetries, and topology, Phys. Rev. D 77 (2008) 015017 [arXiv:0710.3490] [InSPIRE].

[51] G.C. Branco, P.M. Ferreira, L. Lavoura, M.N. Rebelo, M. Sher and J.P. Silva, Theory and phenomenology of two-Higgs-doublet models, Phys. Rept. 516 (2012) 1 [arXiv:1106.0034] [INSPIRE].

[52] R.A. Battye, G.D. Brawn and A. Pilaftsis, Vacuum topology of the two Higgs doublet model, JHEP 08 (2011) 020 [arXiv: 1106.3482] [INSPIRE].

[53] B. Grzadkowski, O.M. Ogreid and P. Osland, Diagnosing CP properties of the 2HDM, JHEP 01 (2014) 105 [arXiv: 1309.6229] [INSPIRE].

[54] B. Grzadkowski, O.M. Ogreid and P. Osland, Natural multi-Higgs model with dark matter and CP-violation, Phys. Rev. D 80 (2009) 055013 [arXiv:0904.2173] [INSPIRE].

[55] A. Arhrib, Unitarity constraints on scalar parameters of the standard and two Higgs doublets model, in the proceedings of the Workshop on Noncommutative Geometry, Superstrings and Particle Physics, June 16-17, Rabat, Morocco (2000) [hep-ph/0012353] [INSPIRE].

[56] S. Kanemura and K. Yagyu, Unitarity bound in the most general two Higgs doublet model, Phys. Lett. B 751 (2015) 289 [arXiv:1509.06060] [INSPIRE].

[57] G. Brawn, Symmetries and topological defects of the two Higgs doublet model, Ph.D., University of Manchester, Machester U.K. (2011).

[58] C.L. Wainwright, CosmoTransitions: computing cosmological phase transition temperatures and bubble profiles with multiple fields, Comput. Phys. Commun. 183 (2012) 2006 [arXiv:1109.4189] [INSPIRE].

[59] D. Stauffer, Scaling theory of percolation clusters, Phys. Rept. 54 (1979) 1 [InSPIRE].

[60] M. Kawasaki, K. Kohri and T. Moroi, Hadronic decay of late-decaying particles and Big-Bang Nucleosynthesis, Phys. Lett. B 625 (2005) 7 [astro-ph/0402490] [INSPIRE].

[61] M. Kawasaki, K. Kohri and T. Moroi, Big-Bang nucleosynthesis and hadronic decay of long-lived massive particles, Phys. Rev. D 71 (2005) 083502 [astro-ph/0408426] [INSPIRE].

[62] T. Hiramatsu, M. Kawasaki and K. Saikawa, On the estimation of gravitational wave spectrum from cosmic domain walls, JCAP 02 (2014) 031 [arXiv:1309.5001] [INSPIRE].

[63] M. Gleiser and R. Roberts, Gravitational waves from collapsing vacuum domains, Phys. Rev. Lett. 81 (1998) 5497 [astro-ph/9807260] [INSPIRE]. 
[64] F. Hoogeveen, The standard model prediction for the electric dipole moment of the electron, Nucl. Phys. B 341 (1990) 322 [inSPIRE].

[65] M.E. Pospelov and I.B. Khriplovich, Electric dipole moment of the $W$ boson and the electron in the Kobayashi-Maskawa model, Sov. J. Nucl. Phys. 53 (1991) 638 [Yad. Fiz. 53 (1991) 1030] [INSPIRE].

[66] M. Pospelov and A. Ritz, CKM benchmarks for electron electric dipole moment experiments, Phys. Rev. D 89 (2014) 056006 [arXiv:1311.5537] [INSPIRE].

[67] Y. Yamaguchi and N. Yamanaka, Quark level and hadronic contributions to the electric dipole moment of charged leptons in the standard model, arXiv:2006.00281 [INSPIRE].

[68] S.M. Barr and A. Zee, Electric dipole moment of the electron and of the neutron, Phys. Rev. Lett. 65 (1990) 21 [Erratum ibid. 65 (1990) 2920] [INSPIRE].

[69] H. La, Vortex solutions in two Higgs systems and tan Beta, hep-ph/9302220 [INSPIRE].

[70] M.A. Earnshaw and M. James, Stability of two doublet electroweak strings, Phys. Rev. D 48 (1993) 5818 [hep-ph/9308223] [INSPIRE].

[71] G. Bimonte and G. Lozano, Vortex solutions in two Higgs doublet systems, Phys. Lett. B 326 (1994) 270 [hep-ph/9401313] [INSPIRE].

[72] M. Eto, Y. Hamada, M. Kurachi and M. Nitta, Topological Nambu monopole in two Higgs doublet models, Phys. Lett. B 802 (2020) 135220 [arXiv: 1904.09269].

[73] M. Eto, Y. Hamada, M. Kurachi and M. Nitta, Dynamics of Nambu monopole in two Higgs doublet models. Cosmological Monopole Collider, JHEP 07 (2020) 004 [arXiv:2003.08772] [INSPIRE].

[74] V.A. Rubakov, Superheavy Magnetic Monopoles and Proton Decay, JETP Lett. 33 (1981) 644 [Pisma Zh. Eksp. Teor. Fiz. 33 (1981) 658] [InSPIRE].

[75] C.G. Callan Jr., Dyon-Fermion dynamics, Phys. Rev. D 26 (1982) 2058 [InSPIRE].

[76] C.G. Callan Jr., Monopole catalysis of baryon decay, Nucl. Phys. B 212 (1983) 391 [INSPIRE].

[77] J.M. Cline and K. Kainulainen, Electroweak baryogenesis at high bubble wall velocities, Phys. Rev. D 101 (2020) 063525 [arXiv: 2001.00568] [InSPIRE].

[78] R. Zhou and L. Bian, Baryon asymmetry and detectable gravitational waves from electroweak phase transition, arXiv:2001.01237 [INSPIRE].

[79] K.-P. Xie, Y. Wu and L. Bian, Electroweak baryogenesis and gravitational waves in a composite Higgs model with high dimensional fermion representations, arXiv:2005.13552 [INSPIRE].

[80] A. Wahab El Kaffas, P. Osland and O.M. Ogreid, Constraining the two-Higgs-doublet-model parameter space, Phys. Rev. D 76 (2007) 095001 [arXiv: 0706.2997] [InSPIRE].

[81] W. Khater and P. Osland, CP violation in top quark production at the LHC and two Higgs doublet models, Nucl. Phys. B 661 (2003) 209 [hep-ph/0302004] [INSPIRE].

[82] P.S. Bhupal Dev, A. Djouadi, R.M. Godbole, M.M. Muhlleitner and S.D. Rindani, Determining the CP properties of the Higgs boson, Phys. Rev. Lett. 100 (2008) 051801 [arXiv: 0707.2878] [INSPIRE].

[83] R. Harnik, A. Martin, T. Okui, R. Primulando and F. Yu, Measuring CP-violation in $h \rightarrow \tau^{+} \tau^{-}$at colliders, Phys. Rev. D 88 (2013) 076009 [arXiv:1308.1094] [InSPIRE]. 
[84] S. Berge, W. Bernreuther and H. Spiesberger, Higgs CP properties using the $\tau$ decay modes at the ILC, Phys. Lett. B $\mathbf{7 2 7}$ (2013) 488 [arXiv: 1308.2674] [InSPIRE].

[85] J. Brod, U. Haisch and J. Zupan, Constraints on CP-violating Higgs couplings to the third generation, JHEP 11 (2013) 180 [arXiv:1310.1385] [INSPIRE].

[86] A. Askew, P. Jaiswal, T. Okui, H.B. Prosper and N. Sato, Prospect for measuring the CP

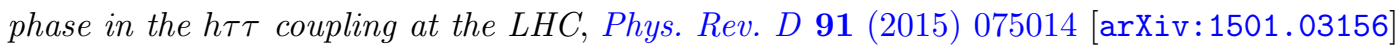
[INSPIRE].

[87] G. Li, H.-R. Wang and S.-h. Zhu, Probing CP-violating httt coupling in $e^{+} e^{-} \rightarrow h \gamma$, Phys. Rev. D 93 (2016) 055038 [arXiv: 1506. 06453] [INSPIRE].

[88] M.R. Buckley and D. Goncalves, Boosting the direct CP measurement of the Higgs-top coupling, Phys. Rev. Lett. 116 (2016) 091801 [arXiv: 1507.07926] [INSPIRE].

[89] S. Berge, W. Bernreuther and S. Kirchner, Prospects of constraining the Higgs boson's CP nature in the tau decay channel at the LHC, Phys. Rev. D 92 (2015) 096012 [arXiv: 1510.03850] [INSPIRE].

[90] T. Han, S. Mukhopadhyay, B. Mukhopadhyaya and Y. Wu, Measuring the CP property of Higgs coupling to tau leptons in the VBF channel at the LHC, JHEP 05 (2017) 128 [arXiv: 1612.00413] [INSPIRE].

[91] K. Hagiwara, K. Ma and H. Yokoya, Probing CP-violation in $e^{+} e^{-}$production of the Higgs boson and toponia, JHEP 06 (2016) 048 [arXiv: 1602.00684] [INSPIRE].

[92] S.D. Rindani, P. Sharma and A. Shivaji, Unraveling the CP phase of top-Higgs coupling in associated production at the LHC, Phys. Lett. B 761 (2016) 25 [arXiv:1605.03806] [INSPIRE].

[93] X. Chen and Y. Wu, Search for CP-violation effects in the $h \rightarrow \tau \tau$ decay with $e^{+} e^{-}$colliders, Eur. Phys. J. C 77 (2017) 697 [arXiv:1703.04855] [inSPIRE].

[94] X. Chen and Y. Wu, Probing the CP-violation effects in the $h \tau \tau$ coupling at the LHC, Phys. Lett. B 790 (2019) 332 [arXiv: 1708.02882] [INSPIRE].

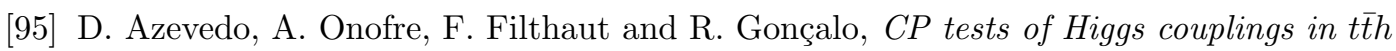
semileptonic events at the LHC, Phys. Rev. D 98 (2018) 033004 [arXiv:1711.05292] [INSPIRE].

[96] K. Hagiwara, H. Yokoya and Y.-J. Zheng, Probing the CP properties of top Yukawa coupling at an $e^{+} e^{-}$collider, JHEP 02 (2018) 180 [arXiv:1712.09953] [INSPIRE].

[97] D. Gonçalves, K. Kong and J.H. Kim, Probing the top-Higgs Yukawa CP structure in dileptonic tth with $M_{2}$-assisted reconstruction, JHEP 06 (2018) 079 [arXiv: 1804.05874] [INSPIRE].

[98] K. Ma, enhancing CP measurement of the Yukawa interactions of top-quark at $e^{-} e^{+}$collider, Phys. Lett. B 797 (2019) 134928 [arXiv:1809.07127].

[99] D.A. Faroughy, J.F. Kamenik, N. Košnik and A. Smolkovič, Probing the CP nature of the top quark Yukawa at hadron colliders, JHEP 02 (2020) 085 [arXiv:1909.00007] [INSPIRE]. 